\title{
Nonlinear Dynamics of Avian Influenza Epidemic Models*
}

\author{
Sanhong LiU ${ }^{1,2}$, Shigui Ruan ${ }^{2,3, \dagger}$ And Xinan Zhang ${ }^{2}$ \\ ${ }^{1}$ School of Mathematics and Statistics, \\ Hubei University of Science and Technology, Xianning 437100, China \\ ${ }^{2}$ School of Mathematics and Statistics, Central China Normal University, Wuhan 430079, China \\ ${ }^{3}$ Department of Mathematics, University of Miami, Coral Gables, FL 33146, USA
}

November 17, 2016

Dedicated to our friend Dr. Dingbian Qian, Professor in the School of Mathematical Sciences at Soochow University, Suzhou, Jiangsu Province, China, who was critically infected by the H7N9 avian influenza virus in April 2013, fearfully stayed in the intensive care unit for more than two months, and miraculously recovered.

\begin{abstract}
Avian influenza is a zoonotic disease caused by the transmission of the avian influenza A virus, such as $\mathrm{H} 5 \mathrm{~N} 1$ and H7N9, from birds to humans. The avian influenza A H5N1 virus has caused more than 500 human infections worldwide with nearly a $60 \%$ death rate since it was first reported in Hong Kong in 1997. The four outbreaks of the avian influenza A H7N9 in China from March 2013 to June 2016 have resulted in 580 human cases including 202 deaths with a death rate of nearly $35 \%$. In this paper, we construct two avian influenza bird-to-human transmission models with different growth laws of the avian population, one with logistic growth and the other with Allee effect, and analyze their dynamical behavior. We obtain the threshold value for the prevalence of avian influenza and investigate the local or global asymptotical stability of each equilibrium of these systems by using linear analysis technique or combining Liapunov function method and LaSalle's invariance principle, respectively. Moreover, we give necessary and sufficient conditions for the occurrence of periodic solutions in the avian influenza system with Allee effect of the avian population. Numerical simulations are also presented to illustrate the theoretical results.

Keywords: avian influenza; Liapunov function; global asymptotical stability; Allee effect; periodic solution
\end{abstract}

\section{Introduction}

Influenza $\mathrm{A}$ viruses are divided into subtypes based on two proteins on the surface of the virus: hemagglutinin (HA) and neuraminidase (NA). For example, the avian influenza A virus designation of H7N9 identifies it as having HA of the H7 subtype and NA of the N9 subtype (CDC [8]). Avian influenza A $\mathrm{H} 7$ viruses are a group of influenza viruses that normally circulate among birds. H7 influenza infections in humans are uncommon, but have been confirmed world-wide in people who have direct contact with infected birds. Most infections have been mild involving only conjunctivitis and mild upper respiratory symptoms (CIDRAP [9] and OIE [59]). Although some H7 viruses (e.g. H7N2, H7N3 and H7N7) have occasionally been found to infect humans, H7N9 had previously been

\footnotetext{
${ }^{*}$ This work was partially supported by the National Natural Science Foundation (NNSF) of China (No.11371161 and No.11228104), the National Science Foundation (DMS-1412454), and a Startup Research Grant from Hubei University of Science and Technology (No. BK1513).

${ }^{\dagger}$ Corresponding author. E-mail address: ruan@math.miami.edu.
} 
isolated only in birds, with outbreaks reported in the Netherlands, Japan, and the United States. Until the 2013 outbreak in China, no human infections with H7N9 viruses had ever been reported (CIDRAP [9] and OIE [59]).

Differing from the highly pathogenic avian influenza virus H5N1, the H7N9 virus does not induce clinical signs in poultry and is classified as a low pathogenicity avian influenza virus (LPAIV) (Pantin-Jackwood et al. [46]). However, the virus can infect humans and most of the reported cases of human H7N9 infection have resulted in severe respiratory illness (Li et al. [39]). From March 31 to August 31, 2013, 134 cases had been reported in mainland China, resulting in 45 deaths(NHFPC [45]), an unusually high rate for a new infection and high death rate. Genetic characterization of H7N9 shows that the virus resulted from the recombination of genes between several parent viruses noted in poultry and wild birds in Asia (Koopmans and De Jong [37]). Evidence suggests that the gene that codes for HA has its origin in ducks and the gene that codes for NA has its origin with ducks and probably also wild birds. The HA genes were circulating in the East Asian flyway in both wild birds and ducks, while the NA genes were introduced from European lineages and transferred to ducks in China by wild birds through migration along the East Asian flyway (Liu et al. [40]). There is very little information on the H7N9 virus in wild birds to access their potential as source of domestic poultry and human infection. The mode of H7N9 virus transmission between avian species remains unknown, but various wild birds have been implicated as a source of transmission. Jones et al. [31] showed that society finches, zebra finches, sparrows, and parakeets are susceptible to H7N9 virus and shed virus into water. Jones et al. [32] further demonstrated that interspecies transmission of H7N9 virus occurs readily between society finches and bobwhite quail but only sporadically between finches and chickens, and transmission occurs through shared water. Since the experimental data of Pantin-Jackwood et al. [46] showed that quail and chickens are susceptible to infection, shed large amounts of virus, and are likely important in the spread of the virus to humans, it is therefore conceivable that passerine birds may serve as vectors for transmission of H7N9 virus to domestic poultry (Jones et al. [32]). Data indicate that the novel avian influenza A H7N9 virus was most likely transmitted from the secondary wholesale market to the retail live-poultry market and then to humans (Bao et al. [4], Chen et al. [11]). To control the outbreak, from late April to early June in 2013, local authorities of the provinces and municipalities, such as Jiangsu, Shanghai, and Zhejiang, temporarily closed the retail live-poultry markets which proved to be an effective control measure. There were no reported cases in the summer and fall 2013. However, the virus came back in November 2013 and again in November 2014 and November 2015. In fact, the second outbreak (from November 2013 to May 2014), the third outbreak (from November 2014 to June 2015), and the fourth outbreak (from November 2015 to June 2016) caused 130 human cases with 35 deaths, 216 confirmed human cases with 99 deaths, and 110 confirmed human cases with 44 deaths, respectively (NHFPC [45]).

Mathematical modeling has become an important tool in analyzing the epidemiological characteristics of infectious diseases and can provide useful control measures (Anderson and May [3], Keeling and Rohani [36]). In 2007, Iwami et al. [28] proposed ordinary differential equation (ODE) models to characterize the dynamical behavior of avian influenza between human and avian populations. Since then various models have been used to study different aspects of avian influenza transmitted by the H5N1 virus. Lucchetti et al. [43] developed an ODE model to describe the transmission dynamics of the avian influenza A virus from birds to humans and used the model to fit the human cases reported by the WHO. Iwami et al. [29] investigated relations between the evolution of virulence and the effectiveness of pandemic control measures after the emergence of mutant avian influenza. Jung et al. [33] extended the study of Iwami et al. [28] for the prevention of the pandemic influenza to evaluate the time-dependent optimal prevention policies, which were associated with elimination policy and quarantine policy, considering its execution cost. Iwami et al. [30] designed and analyzed a deterministic patch-structured model in heterogeneous areas (with or without vaccination) illustrating transmission of vaccine-sensitive and vaccine-resistant strains during a vaccination program. Gumel [24] incorporated the dynamics of both wild and domestic birds and the isolation of individuals with symptoms of both the avian and mutant strains. Ma 
and Wang [44] formulated a discrete-time model with reproductive and overwintering periods to assess the impact of avian influenza transmission in poultry. Bourouiba et al. [5] investigated the role of migratory birds in the spread of $\mathrm{H} 5 \mathrm{~N} 1$ avian influenza among birds by considering a system of delay differential equations for the numbers of birds on patches, where the delays represent the flight times between patches. See also Gourley et al. [22]. Tuncer and Martcheva [52] constructed several bird-to-human transmission models to investigate the mechanisms for the seasonality in avian influenza $\mathrm{H} 5 \mathrm{~N} 1$ transmission. Wang and $\mathrm{Wu}$ [55] constructed a periodic systems of delay differential equations modeling the spread of avian influenza by migratory birds between the refuge ground and the summer breeding site. Chong and Smith [12] proposed two Filippov models with threshold policy to determine culling of infected birds and quarantine.

Considering the fact that the domesticated birds are probably the important infectious source for human population, Iwami et al. [28] assumed that the avian populations are subject to the rule of constant growth. But the possibility that migrant birds are viewed as the original infection source is the largest (Zhang et al. [62]). Migratory hosts may transmit pathogens to new areas, leading to the exposure and potential infection of new host species (Altizer et al. [1]). Resident hosts, immunologically naive to these novel pathogens, may subsequently act as local amplifiers. For example, the global spread of West Nile Virus is considered to be greatly facilitated by migratory birds introducing the virus to other wildlife and humans in many parts of the world (Rappole [47]). It is well-known that the logistic growth, where the rate of reproduction is proportional to both the existing population and the amount of available resources and increases quickly at first and then more slowly as the population approaches its carrying capacity, is more reasonable than the constant growth for the wildlife birds, including migratory and resident birds. Allee effect, a phenomenon in which the reproduction rate of a population decreases when its density drops below a certain critical level, was firstly observed by Allee [2] about aggregation and associated cooperative and social characteristics among members of a species in animal populations. The phenomenon in biology is called strong Allee effect, which is particularly relevant to endangered species and small or invasive populations. Habitat destruction, spread of alien species, overharvest, pollution (including siltation), and disease (caused by either alien or native pathogens) are responsible for endangering species (Wilcove et. al. [57]). The study of Serrano et al. [49] on Allee effect in colonial birds demonstrates that Allee effect, that is positive density dependence, appears to be the cause of the evolution of dispersal behavior. Skagen and Yackel [50] observed that population density of small bird populations is correlated positively with both per capita fecundity and population growth rate due to the Allee effect.

It has been reported that some wild species, such as the African wild dog Lycaon pictus (Burrows et al. [6]) and the island fox Urocyon littoralis (Clifford et al. [13]), suffer from both disease and an Allee effect. Diseases can drive populations to low densities as a result of Allee effect, in particular for diseases having reservoirs or affecting populations that are at small preepidemic sizes (de Castro and Bolker [18]) or for native island species exposed to new pathogens (Wikelski et al. [56]). In wild populations of Serins (Serinus serinus), Senar and Conroy [48] reported that avian pox infections were very virulent and survival rates of infected birds were half that of uninfected ones. Recently, great attention has been paid to the theoretical modeling and analysis of the joint interplay of infectious disease and Allee effects (see Hilker et al. [26,27], Thieme et al. [51], Friedman and Yakubu [20,21], Kang and Castillo-Chavez [34,35], and the references cited therein). On one hand, it has been observed that recurrent infectious disease outbreaks tend to enhance the deleterious role of Allee effects within diseases capable of inducing reductions in host fitness (Kang and Castillo-Chavez [35]). On the other hand, sustained oscillations can occur induced by Allee effects via bifurcations (Hilker et al. [26], Thieme et al. [51], Cai et al. [7], Kang and Castillo-Chavez [35]).

In this paper we construct two simplified avian-human epidemic models according to different growth rates of the avian population, namely, with avian population being subject to logistic growth and Allee effect. We always assume that the avian influenza virus does not spread from person to person and mutate. The avian population is classified into two subclasses: susceptible 
and infective, denoted by $S_{a}(t)$ and $I_{a}(t)$, respectively, and the human population is classified into three subclasses: susceptible, infective and recovered/removed, denoted by $S_{h}(t), I_{h}(t)$, and $R_{h}(t)$, respectively. In order to construct the corresponding model, we make the following assumptions:

(1) The net growth rate of the susceptible avian population is described by the function $g\left(S_{a}\right)$, where $g(\cdot): \mathbb{R}_{+} \rightarrow \mathbb{R}$ is continuous, $\mathbb{R}=(-\infty, \infty), \mathbb{R}_{+}=[0, \infty)$;

(2) All new recruitments and newborns of the human population are susceptible, the rate is denoted by $\Pi_{h}$;

(3) The avian influenza virus is not contagious from an infective human to a susceptible human. It is only contagious from an infective avian to a susceptible human;

(4) An infected avian keeps in the state of disease and cannot recover, but an infected human can recover and the recovered human has permanent immunity;

(5) The incidence rate between the susceptible avian and the infective avian is bilinear. The incidence rate between the susceptible human and the infective avian is also bilinear.

Based on the above assumptions, we have the following SI-SIR avian influenza model:

$$
\left\{\begin{array}{l}
\frac{d S_{a}}{d t}=g\left(S_{a}\right)-\beta_{a} S_{a} I_{a} \\
\frac{d I_{a}}{d t}=\beta_{a} S_{a} I_{a}-\left(\mu_{a}+\delta_{a}\right) I_{a} \\
\frac{d S_{h}}{d t}=\Pi_{h}-\beta_{h} S_{h} I_{a}-\mu_{h} S_{h} \\
\frac{d I_{h}}{d t}=\beta_{h} S_{h} I_{a}-\left(\mu_{h}+\delta_{h}+\gamma\right) I_{h} \\
\frac{d R_{h}}{d t}=\gamma I_{h}-\mu_{h} R_{h},
\end{array}\right.
$$

where $\beta_{a}$ is the transmission rate from infective avian to susceptible avian, $\mu_{a}$ is the natural death rate of the avian population, $\delta_{a}$ is the disease-related death rate of the infected avian; $\beta_{h}$ is the transmission rate from the infective avian to the susceptible human, $\mu_{h}$ is the natural death rate of the human population; $\delta_{h}$ is the disease-related death rate of the infected human; $\gamma$ is the recovery rate of the infective human. If the susceptible avian population is subject to the logistic growth, then

$$
g\left(S_{a}\right)=r_{a} S_{a}\left(1-\frac{S_{a}}{K_{a}}\right)
$$

where $r_{a}$ and $K_{a}$ are the intrinsic growth rate and maximal carrying capacity of the avian population, respectively. If the susceptible avian population is subject to Allee effect, then

$$
g\left(S_{a}\right)=r_{a} S_{a}\left(1-\frac{S_{a}}{M_{a}}\right)\left(\frac{S_{a}}{m_{a}}-1\right),
$$

where $r_{a}, M_{a}$, and $m_{a}\left(m_{a}<M_{a}\right)$ are the intrinsic growth rate, the maximal carrying capacity and the critical carrying capacity of the avian population, respectively. We assume that all parameters are positive.

We will analyze the global asymptotical stability of these systems and compare the sizes of the basic reproduction numbers for both cases. The paper is organized as follows. The global analysis of avian-human epidemic models in which the avian population is subject to the rule of logistic growth law and Allee effect is discussed in Sections 2 and 3, respectively, where the human population is always subject to the rule of constant growth. In Section 4, we compare the sizes of two basic reproduction numbers and provide numerical simulations of the model for both cases. A brief discussion about the biological interpretation and conclusion is given in the last section. 


\section{Model (1) with logistic growth for avian population}

\subsection{The model}

If the net growth rate of the avian population is subject to the logistic growth law in system (1), then we obtain the following SI-SIR model:

$$
\left\{\begin{array}{l}
\frac{d S_{a}}{d t}=r_{a} S_{a}\left(1-\frac{S_{a}}{K_{a}}\right)-\beta_{a} S_{a} I_{a} \\
\frac{d I_{a}}{d t}=\beta_{a} S_{a} I_{a}-\left(\mu_{a}+\delta_{a}\right) I_{a} \\
\frac{d S_{h}}{d t}=\Pi_{h}-\beta_{h} I_{a} S_{h}-\mu_{h} S_{h} \\
\frac{d I_{h}}{d t}=\beta_{h} I_{a} S_{h}-\left(\mu_{h}+\delta_{h}+\gamma\right) I_{h} \\
\frac{d R_{h}}{d t}=\gamma I_{h}-\mu_{h} R_{h},
\end{array}\right.
$$

where $r_{a}\left(K_{a}\right)$ is the intrinsic growth rate (the maximal carrying capacity) of the avian population, the other assumptions and the meanings of the other parameters are the same as in (1). System (4) has a unique solution satisfying initial conditions in $\mathbb{R}_{+}^{5}$ which is the positively invariant set for system (4).

We can deduce two disease-free equilibria given by $A\left(0,0, S_{h}^{*}, 0,0\right)$ and $B\left(K_{a}, 0, S_{h}^{*}, 0,0\right)$ from system (4), where $S_{h}^{*}=\frac{\Pi_{h}}{\mu_{h}}$.

Following the definition and computation procedure in Diekmann et al. [19] and van den Driessche and Watmough [54], we can rewrite system (4) as follows:

$$
\frac{d X}{d t}=\mathscr{F}-\mathscr{V}
$$

where,

$$
X(t)=\left(\begin{array}{c}
I_{a}(t) \\
I_{h}(t) \\
S_{a}(t) \\
S_{h}(t) \\
R_{h}(t)
\end{array}\right), \mathscr{F}=\left(\begin{array}{c}
\beta_{a} I_{a} S_{a} \\
\beta_{h} I_{a} S_{h} \\
0 \\
0 \\
0
\end{array}\right), \quad \mathscr{V}=\left(\begin{array}{c}
\left(\mu_{a}+\delta_{a}\right) I_{a} \\
\left(\mu_{h}+\delta_{h}+\gamma\right) I_{h} \\
\beta_{a} I_{a} S_{a}-\beta_{a} S_{a}\left(1-\frac{S_{a}}{K_{a}}\right) \\
\mu_{h} S_{h}+\beta_{h} I_{a} S_{h}-\Pi_{h} \\
\mu_{h} R_{h}-\gamma I_{h}
\end{array}\right)
$$

then,

$$
F=\left(\begin{array}{cc}
\beta_{a} K_{a} & 0 \\
\beta_{h} S_{h}^{*} & 0
\end{array}\right), V=\left(\begin{array}{cc}
\mu_{a}+\delta_{a} & 0 \\
0 & \mu_{h}+\delta_{h}+\gamma
\end{array}\right), \quad F V^{-1}=\left(\begin{array}{cc}
\frac{\beta_{a} K_{a}}{\mu_{a}+\delta_{a}} & 0 \\
\frac{\beta_{h} S_{h}^{*}}{\mu_{a}+\delta_{a}} & 0
\end{array}\right) .
$$

Hence, we derive the basic reproduction number as follows

$$
\mathcal{R}_{0,1}=\frac{K_{a} \beta_{a}}{\mu_{a}+\delta_{a}} .
$$

If $\mathcal{R}_{0,1}>1$, we can also derive a unique endemic equilibrium given by $C\left(S_{a}^{* *}, I_{a}^{* *}, S_{h}^{* *}, I_{h}^{* *}, R_{h}^{* *}\right)$, where

$$
\begin{aligned}
& S_{a}^{* *}=\frac{\mu_{a}+\delta_{a}}{\beta_{a}}, \quad I_{a}^{* *}=\frac{r_{a}\left(\mu_{a}+\delta_{a}\right)}{K_{a} \beta_{a}^{2}}\left(\mathcal{R}_{0,1}-1\right), \\
& S_{h}^{* *}=\frac{\Pi_{h}}{\beta_{h} I_{a}^{* *}+\mu_{h}}, \quad I_{h}^{* *}=\frac{\beta_{h} I_{a}^{* *} S_{h}^{* *}}{\mu_{h}+\delta_{h}+\gamma}, \quad R_{h}^{* *}=\frac{\gamma I_{h}^{* *}}{\mu_{h}} .
\end{aligned}
$$

Before analyzing the dynamical behavior of the full model (4), we study the dynamical behavior of the avian-only subsystem. 


\subsection{Analysis of the avian-only subsystem}

Consider the avian-only subsystem, given by the first two equations of system (4), as follows:

$$
\left\{\begin{array}{l}
\frac{d S_{a}}{d t}=r_{a} S_{a}\left(1-\frac{S_{a}}{K_{a}}\right)-\beta_{a} S_{a} I_{a} \\
\frac{d I_{a}}{d t}=\beta_{a} S_{a} I_{a}-\left(\mu_{a}+\delta_{a}\right) I_{a} .
\end{array}\right.
$$

It should be noted that the above avian system is independent of the human system. Clearly, $\mathbb{R}_{+}^{2}$ is the positively invariant attracting set of subsystem (5). Next we will discuss the dynamical behavior of solutions to subsystem (5) in $\mathbb{R}_{+}^{2}$.

\subsubsection{Local stability of the avian-only subsystem (5)}

The avian-only subsystem (5) always has two disease-free equilibria given by $A_{a}(0,0)$ and $B_{a}\left(K_{a}, 0\right)$. If $\mathcal{R}_{0,1}>1$, the system also has a unique endemic equilibrium given by $C_{a}\left(S_{a}^{* *}, I_{a}^{* *}\right)$.

Lemma 2.1. (i) The disease-free equilibrium $A_{a}$ is always unstable; (ii) If $\frac{\mu_{a}+\delta_{a}}{\beta_{a}} \geq K_{a}$ (i.e., $\left.\mathcal{R}_{0,1} \leq 1\right)$, then the disease-free equilibrium $B_{a}$ is locally asymptotically stable for positive trajectories; (iii) If $0<\frac{\mu_{a}+\delta_{a}}{\beta_{a}}<K_{a}$ (i.e., $\mathcal{R}_{0,1}>1$ ), then the disease-free equilibrium $B_{a}$ is unstable but the endemic equilibrium $C_{a}$ is locally asymptotically stable.

Proof. The characteristic equation of the Jacobian matrix at an arbitrary equilibrium $\left(S_{a}, I_{a}\right)$ is

$$
\left(\lambda-\left(r_{a}-\frac{2 r_{a}}{K_{a}} S_{a}-\beta_{a} I_{a}\right)\right)\left(\lambda-\left(\beta_{a} S_{a}-\mu_{a}-\delta_{a}\right)\right)+\beta_{a}^{2} S_{a} I_{a}=0 .
$$

(i) If $\left(S_{a}, I_{a}\right)=(0,0)$, the eigenvalues are $\lambda_{1}=r_{a}>0, \lambda_{2}=-\left(\mu_{a}+\delta_{a}\right)<0$. Hence, the equilibrium $A_{a}$ is always unstable.

(ii) If $\mathcal{R}_{0,1}<1$ and $\left(S_{a}, I_{a}\right)=\left(K_{a}, 0\right)$, the eigenvalues are $\lambda_{1}=-r_{a}<0, \lambda_{2}=\left(\mu_{a}+\delta_{a}\right)\left(\mathcal{R}_{0,1}-\right.$ 1) $<0$. Hence, the equilibrium $B_{a}$ is locally asymptotically stable.

(iii) If $\mathcal{R}_{0,1}>1$ and $\left(S_{a}, I_{a}\right)=\left(K_{a}, 0\right)$, the eigenvalues are $\lambda_{1}=-r_{a}<0, \lambda_{2}=\left(\mu_{a}+\delta_{a}\right)\left(\mathcal{R}_{0,1}-\right.$ $1)>0$. Hence, the equilibrium $B_{a}$ is unstable; If $\mathcal{R}_{0,1}>1$ and $\left(S_{a}, I_{a}\right)=\left(S_{a}^{* *}, I_{a}^{* *}\right)$, the above characteristic equation becomes

$$
\lambda^{2}+\frac{r_{a}}{K_{a}} S_{a}^{* *} \lambda+\beta_{a}^{2} S_{a}^{* *} I_{a}^{* *}=0 .
$$

Since $S_{a}^{* *}>0$ and $I_{a}^{* *}>0$ if $\mathcal{R}_{0,1}>1$, all eigenvalues have negative real parts. Hence, the equilibrium $C_{a}$ is locally asymptotically stable.

Remark 2.2. If $\mathcal{R}_{0,1}=1$, then the endemic equilibrium $C_{a}$ coincides with the disease-free equilibrium $B_{a}$ which is a saddle-node and is locally asymptotically stable for positive trajectories.

\subsubsection{Global stability of the avian-only subsystem (5)}

Lemma 2.3. (i) If $\frac{\mu_{a}+\delta_{a}}{\beta_{a}} \geq K_{a}$ (i.e., $\mathcal{R}_{0,1} \leq 1$ ), then the disease-free equilibrium $B_{a}$ is globally asymptotically stable for positive trajectories; (ii) If $0<\frac{\mu_{a}+\delta_{a}}{\beta_{a}}<K_{a}$ (i.e., $\mathcal{R}_{0,1}>1$ ), then the endemic equilibrium $C_{a}$ is globally asymptotically stable.

Proof. (i) If $\mathcal{R}_{0,1} \leq 1$, we choose a Liapunov function as follows

$$
V_{1}=S_{a}-K_{a}-K_{a} \ln \frac{S_{a}}{K_{a}}+I_{a}
$$


Then we have

$$
\begin{aligned}
\left.\frac{d V_{1}}{d t}\right|_{(5)} & =\left(S_{a}-K_{a}\right)\left(r_{a}-\frac{r_{a} S_{a}}{K_{a}}-\beta_{a} I_{a}\right)+\beta_{a} S_{a} I_{a}-\left(\mu_{a}+\delta_{a}\right) I_{a} \\
& =\frac{-r_{a}\left(S_{a}-K_{a}\right)^{2}}{K_{a}}-\beta_{a} I_{a}\left(S_{a}-K_{a}\right)+\beta_{a} S_{a} I_{a}-\left(\mu_{a}+\delta_{a}\right) I_{a} \\
& =\frac{-r_{a}\left(S_{a}-K_{a}\right)^{2}}{K_{a}}+\beta_{a} K_{a} I_{a}-\left(\mu_{a}+\delta_{a}\right) I_{a} \\
& =\frac{-r_{a}\left(S_{a}-K_{a}\right)^{2}}{K_{a}}+I_{a}\left(\mu_{a}+\delta_{a}\right)\left(\mathcal{R}_{0,1}-1\right) \leq 0 .
\end{aligned}
$$

Since $\left\{\left(S_{a}, I_{a}\right) \in \mathbb{R}_{+}^{2}: \frac{d V_{1}}{d t}=0\right\}=\left\{\left(S_{a}, I_{a}\right) \in \mathbb{R}_{+}^{2}: S_{a}=K_{a}, I_{a}=0\right\}=\left\{B_{a}\right\}$, according to LaSalle's invariance principle (Hale [25]), the equilibrium $B_{a}$ is globally asymptotically stable for positive trajectories.

(ii) If $\mathcal{R}_{0,1}>1$, we choose a Liapunov function

$$
V_{2}=\left(S_{a}-S_{a}^{* *}-S_{a}^{* *} \ln \frac{S_{a}}{S_{a}^{* *}}\right)+\left(I_{a}-I_{a}^{* *}-I_{a}^{* *} \ln \frac{I_{a}}{I_{a}^{* *}}\right) .
$$

Then we obtain

$$
\begin{aligned}
\left.\frac{d V_{2}}{d t}\right|_{(5)} & =\left(S_{a}-S_{a}^{* *}\right)\left(r_{a}\left(1-\frac{S_{a}}{K_{a}}\right)-\beta_{a} I_{a}\right)+\left(I_{a}-I_{a}^{* *}\right)\left(\beta_{a} S_{a}-\mu_{a}-\delta_{a}\right) \\
& =\left(S_{a}-S_{a}^{* *}\right)\left(\frac{r_{a} S_{a}^{* *}}{K_{a}}+\beta_{a} I_{a}^{* *}-\frac{r_{a} S_{a}}{K_{a}}-\beta_{a} I_{a}\right)+\beta_{a}\left(I_{a}-I_{a}^{* *}\right)\left(S_{a}-S_{a}^{* *}\right) \\
& =-\frac{r_{a}}{K_{a}}\left(S_{a}-S_{a}^{* *}\right)^{2} \leq 0 .
\end{aligned}
$$

It follows that $\hat{D}=\left\{\left(S_{a}, I_{a}\right) \in \operatorname{int} R_{+}^{2}: \frac{d V_{2}}{d t}=0\right\}=\left\{\left(S_{a}, I_{a}\right): S_{a}=S_{a}^{* *}, I_{a} \geq 0\right\}$. If $\hat{D}$ is an invariant set of subsystem (5), then $I_{a}=I_{a}^{* *}$ by the first equation of subsystem (5). Hence $D_{2}=$ $\left\{C_{a}\right\}$. LaSalle's invariance principle implies that the equilibrium $C_{a}$ is globally asymptotically stable.

\subsection{Analysis of the full system}

Since the first four equations of system (4) are independent of the variable $R_{h}$, we only need to analyze the dynamical behavior of the following equivalent system

$$
\left\{\begin{array}{l}
\frac{d S_{a}}{d t}=r_{a} S_{a}\left(1-\frac{S_{a}}{K_{a}}\right)-\beta_{a} S_{a} I_{a} \\
\frac{d I_{a}}{d t}=\beta_{a} S_{a} I_{a}-\left(\mu_{a}+\delta_{a}\right) I_{a} \\
\frac{d S_{h}}{d t}=\Pi_{h}-\beta_{h} I_{a} S_{h}-\mu_{h} S_{h} \\
\frac{d I_{h}}{d t}=\beta_{h} I_{a} S_{h}-\left(\mu_{h}+\delta_{h}+\gamma\right) I_{h} .
\end{array}\right.
$$

Clearly, $\mathbb{R}_{+}^{4}$ is a positively invariant attracting set. We discuss the dynamical behavior of system (6) in the positively invariant set $\mathbb{R}_{+}^{4}$.

\subsubsection{Local stability of the full system (6)}

System (6) always has two disease-free equilibria given by $A_{a h}\left(0,0, S_{h}^{*}, 0\right)$ and $B_{a h}\left(K_{a}, 0, S_{h}^{*}, 0\right)$; if $R_{0,1}>1$, then system (6) also has a unique endemic equilibrium given by $C_{a h}\left(S_{a}^{* *}, I_{a}^{* *}, S_{h}^{* *}, I_{h}^{* *}\right)$. 
Lemma 2.4. (i) The disease-free equilibrium $A_{a h}$ is always unstable; (ii) If $\frac{\mu_{a}+\delta_{a}}{\beta_{a}} \geq K_{a}$ (i.e., $\left.\mathcal{R}_{0,1} \leq 1\right)$, then the disease-free equilibrium $B_{a h}$ is locally asymptotically stable for positive trajectories; (iii) If $\frac{\mu_{a}+\delta_{a}}{\beta_{a}}<K_{a}$ (i.e., $\mathcal{R}_{0,1}>1$ ), then the disease-free equilibrium $B_{a h}$ is unstable and the endemic equilibrium $C_{a h}$ is locally asymptotically stable.

Proof. The characteristic equation of the Jacobian matrix at an arbitrary equilibrium $\left(S_{a}, I_{a}, S_{h}, I_{h}\right)$ takes the form

$$
\left(\lambda+\beta_{h} I_{a}+\mu_{h}\right)\left(\lambda+\mu_{h}+\delta_{h}+\gamma\right)\left(\left(\lambda-\left(r_{a}-\frac{2 r_{a}}{K_{a}} S_{a}-\beta_{a} I_{a}\right)\right)\left(\lambda-\left(\beta_{a} S_{a}-\mu_{a}-\delta_{a}\right)\right)+\beta_{a}^{2} S_{a} I_{a}\right)=0 .
$$

(i) If $\left(S_{a}, I_{a}, S_{h}, I_{h}\right)=\left(0,0, S_{h}^{*}, 0\right)$, the eigenvalues are $\lambda_{1}=r_{a}>0, \lambda_{2}=-\left(\mu_{a}+\delta_{a}\right), \lambda_{3}=-\mu_{h}$, $\lambda_{4}=-\left(\mu_{h}+\delta_{h}+\gamma\right)$. Hence, $A_{a h}$ is always unstable.

(ii) If $\mathcal{R}_{0,1}<1$ and $\left(S_{a}, I_{a}, S_{h}, I_{h}\right)=\left(K_{a}, 0, S_{h}^{*}, 0\right)$, the eigenvalues are $\lambda_{1}=-r_{a}<0, \lambda_{2}=$ $\left(\mu_{a}+\delta_{a}\right)\left(\mathcal{R}_{0,1}-1\right)<0, \lambda_{3}=-\mu_{h}<0, \lambda_{4}=-\left(\mu_{h}+\delta_{h}+\gamma\right)<0$. Hence, the equilibrium $B_{a h}$ is locally asymptotically stable.

(iii) If $\mathcal{R}_{0,1}>1$ and $\left(S_{a}, I_{a}, S_{h}, I_{h}\right)=\left(K_{a}, 0, S_{h}^{*}, 0\right)$, the eigenvalues are $\lambda_{1}=-r_{a}<0, \lambda_{2}=$ $\left(\mu_{a}+\delta_{a}\right)\left(\mathcal{R}_{0,1}-1\right)>0, \lambda_{3}=-\mu_{h}<0, \lambda_{4}=-\left(\mu_{h}+\delta_{h}+\gamma\right)<0$. Hence, the equilibrium $B_{a h}$ is unstable; If $\mathcal{R}_{0,1}>1$ and $\left(S_{a}, I_{a}, S_{h}, I_{h}\right)=\left(S_{a}^{* *}, I_{a}^{* *}, S_{h}^{* *}, I_{h}^{* *}\right)$, the characteristic equation of the Jacobian matrix at the endemic equilibrium $C_{a h}$ is

$$
\left(\lambda^{2}+\frac{r_{a}}{K_{a}} S_{a}^{* *} \lambda+\beta_{a}{ }^{2} S_{a}^{* *} I_{a}^{* *}\right)\left(\lambda+\beta_{h} I_{a}^{* *}+\mu_{h}\right)\left(\lambda+\mu_{h}+\delta_{h}+\gamma\right)=0 .
$$

Since $S_{a}^{* *}>0, I_{a}^{* *}>0$ if $\mathcal{R}_{0,1}>1$, all eigenvalues have negative real parts. Hence, the endemic equilibrium $C_{a h}$ is locally asymptotically stable.

Remark 2.5. If $\mathcal{R}_{0,1}=1$, then the equilibrium $C_{a h}$ coincides with the equilibrium $B_{a h}$ which is a saddle-node and is locally asymptotically stable for positive trajectories.

\subsubsection{Global stability of the full system (6)}

Theorem 2.6. (i) If $\frac{\mu_{a}+\delta_{a}}{\beta_{a}} \geq K_{a}$ (i.e. $\mathcal{R}_{0,1} \leq 1$ ), then the disease-free equilibrium $B_{a h}$ of the full system (6) is globally asymptotically stable; (ii) If $0<\frac{\mu_{a}+\delta_{a}}{\beta_{a}}<K_{a}$ (i.e., $\mathcal{R}_{0,1}>1$ ), then the endemic equilibrium $C_{a h}$ of the full system (6) is globally asymptotically stable.

Proof. (i) According to Lemma 2.3, the disease-free equilibrium $B_{a}$ of the avian-only subsystem (5) is globally asymptotically stable if $\mathcal{R}_{0,1} \leq 1$. To prove the global stability of $B_{a h}$, we only need to consider system (6) with the avian components already at the disease-free steady state, given by

$$
\left\{\begin{array}{l}
\frac{d S_{h}}{d t}=\Pi_{h}-\mu_{h} S_{h} \\
\frac{d I_{h}}{d t}=-\left(\mu_{h}+\delta_{h}+\gamma\right) I_{h} .
\end{array}\right.
$$

Clearly, we can obtain that $S_{h} \rightarrow S_{h}^{*}, I_{h} \rightarrow 0$ if $t \rightarrow \infty$. Hence, the disease-free equilibrium $B_{a h}$ is globally asymptotically stable.

(ii) Similarly, by Lemma 2.3, the endemic equilibrium $C_{a}$ of avian-only subsystem (5) is globally asymptotically stable if $\mathcal{R}_{0,1}>1$. To prove the global stability of the equilibrium $C_{a h}$, we only need to consider system (6) with the avian components already at the endemic steady state, given by

$$
\left\{\begin{array}{l}
\frac{d S_{h}}{d t}=\Pi_{h}-\beta_{h} I_{a}^{* *} S_{h}-\mu_{h} S_{h} \\
\frac{d I_{h}}{d t}=\beta_{h} I_{a}^{* *} S_{h}-\left(\mu_{h}+\delta_{h}+\gamma\right) I_{h} .
\end{array}\right.
$$


We can easily deduce that subsystem (8) has a unique positive equilibrium $\left(S_{h}^{* *}, I_{h}^{* *}\right)$ which is locally asymptotically stable.

To prove the global stability of the positive equilibrium $\left(S_{h}^{* *}, I_{h}^{* *}\right)$ of subsystem (8), we choose a Lyapunov function as follows

$$
V=S_{h}^{* *}\left(\frac{S_{h}}{S_{h}^{* *}}-\ln \frac{S_{h}}{S_{h}^{* *}}\right)+I_{h}^{* *}\left(\frac{I_{h}}{I_{h}^{* *}}-\ln \frac{I_{h}}{I_{h}^{* *}}\right),
$$

then,

$$
\left.\frac{d V}{d t}\right|_{(8)}=\frac{d S_{h}}{d t}-\frac{S_{h}^{* *}}{S_{h}} \frac{d S_{h}}{d t}+\frac{d I_{h}}{d t}-\frac{I_{h}^{* *}}{I_{h}} \frac{d I_{h}}{d t} .
$$

Using the relationships that (at endemic state) $\Pi_{h}=\beta_{h} I_{a}^{* *} S_{h}^{* *}+\mu_{h} S_{h}^{* *}$ and $\mu_{h}+\delta_{h}+\gamma=\frac{\beta_{h} I_{a}^{* *} S_{h}^{* *}}{I_{h}^{* *}}$, we obtain

$$
\begin{aligned}
\frac{d S_{h}}{d t}-\frac{S_{h}^{* *}}{S_{h}} \frac{d S_{h}}{d t}= & \left(\Pi_{h}-\beta_{h} I_{a}^{* *} S_{h}-\mu_{h} S_{h}\right)-\frac{S_{h}^{* *}}{S_{h}}\left(\Pi_{h}-\beta_{h} I_{a}^{* *} S_{h}-\mu_{h} S_{h}\right) \\
= & \left(\beta_{h} I_{a}^{* *} S_{h}^{* *}+\mu_{h} S_{h}^{* *}-\beta_{h} I_{a}^{* *} S_{h}-\mu_{h} S_{h}\right) \\
& -\frac{S_{h}^{* *}}{S_{h}}\left(\beta_{h} I_{a}^{* *} S_{h}^{* *}+\mu_{h} S_{h}^{* *}-\beta_{h} I_{a}^{* *} S_{h}-\mu_{h} S_{h}\right) \\
= & \mu_{h} S_{h}^{* *}\left(2-\frac{S_{h}^{* *}}{S_{h}}-\frac{S_{h}}{S_{h}^{* *}}\right)+2 \beta_{h} I_{a}^{* *} S_{h}^{* *} \\
& -\beta_{h} I_{a}^{* *} S_{h}-\beta_{h} I_{a}^{* *} \frac{\left(S_{h}^{* *}\right)^{2}}{S_{h}}
\end{aligned}
$$

and

$$
\begin{aligned}
\frac{d I_{h}}{d t}-\frac{I_{h}^{* *}}{I_{h}} \frac{d I_{h}}{d t} & =\left(\beta_{h} I_{a}^{* *} S_{h}-\left(\mu_{h}+\delta_{h}+\gamma\right) I_{h}\right)-\frac{I_{h}^{* *}}{I_{h}}\left(\beta_{h} I_{a}^{* *} S_{h}-\left(\mu_{h}+\delta_{h}+\gamma\right) I_{h}\right) \\
& =\left(\beta_{h} I_{a}^{* *} S_{h}-\beta_{h} I_{a}^{* *} S_{h}^{* *} \frac{I_{h}}{I_{h}^{* *}}\right)-\frac{I_{h}^{* *}}{I_{h}}\left(\beta_{h} I_{a}^{* *} S_{h}-\beta_{h} I_{a}^{* *} S_{h}^{* *} \frac{I_{h}}{I_{h}^{* *}}\right) \\
& =\beta_{h} I_{a}^{* *} S_{h}+\beta_{h} I_{a}^{* *} S_{h}^{* *}-\beta_{h} I_{a}^{* *} S_{h}^{* *} \frac{I_{h}}{I_{h}^{* *}}-\beta_{h} I_{a}^{* *} S_{h} \frac{I_{h}^{* *}}{I_{h}}
\end{aligned}
$$

Therefore, we have

$$
\left.\frac{d V}{d t}\right|_{(8)}=\mu_{h} S_{h}^{* *}\left(2-\frac{S_{h}^{* *}}{S_{h}}-\frac{S_{h}}{S_{h}^{* *}}\right)+\beta_{h} I_{a}^{* *} S_{h}^{* *}\left(3-\frac{S_{h}^{* *}}{S_{h}}-\frac{I_{h}}{I_{h}^{* *}}-\frac{S_{h}}{S_{h}^{* *}} \frac{I_{h}^{* *}}{I_{h}}\right) .
$$

Since the arithmetic mean exceeds the geometric mean, we have

$$
\begin{gathered}
2-\frac{S_{h}^{* *}}{S_{h}}-\frac{S_{h}}{S_{h}^{* *}} \leq 0, \\
3-\frac{S_{h}^{* *}}{S_{h}}-\frac{I_{h}}{I_{h}^{* *}}-\frac{S_{h}}{S_{h}^{* *}} \frac{I_{h}^{* *}}{I_{h}} \leq 0 .
\end{gathered}
$$

Hence, $\left.\frac{d V}{d t}\right|_{(8)} \leq 0$. Due to $\tilde{D}=\left\{\left(S_{h}, I_{h}\right) \in \operatorname{int} \mathbb{R}_{+}^{2}: \frac{d V}{d t}=0\right\}=\left\{\left(S_{h}^{* *}, I_{h}^{* *}\right)\right\}$, by the LaSalle's invariance principle, it follows that $S_{h} \rightarrow S_{h}^{* *}$ and $I_{h} \rightarrow I_{h}^{* *}$ if $t \rightarrow \infty$. Therefore, the endemic equilibrium $C_{a h}$ of the full system (6) is globally asymptotically stable.

Now we can state our results for the original SI-SIR model (4) with logistic growth for the avian population.

Corollary 2.7. (i) The disease-free equilibrium A of model (4) with logistic avian growth is always unstable; (ii) If $\frac{\mu_{a}+\delta_{a}}{\beta_{a}} \geq K_{a}$ (i.e., $\mathcal{R}_{0,1} \leq 1$ ), then the disease-free equilibrium $B$ of model (4) is globally asymptotically stable for positive trajectories; (iii) If $0<\frac{\mu_{a}+\delta_{a}}{\beta_{a}}<K_{a}$ (i.e., $\mathcal{R}_{0,1}>1$ ), then the disease-free equilibrium $B$ of model (4) is unstable but the endemic equilibrium $C$ of model (4) is globally asymptotically stable. 
Remark 2.8. If the susceptible avian population is subject to constant growth, that is, $g\left(S_{a}\right)=$ $\Pi_{a}-\mu_{a} S_{a}$, where $\Pi_{a}$ is the recruit rate of new recruitments and newborns and $\mu_{a}$ is the mortality rate of the avian population, then we can obtain analogous results and the dynamics are very much similar to that of system (4) with logistic avian growth.

\section{Model (1) with Allee effect for avian population}

\subsection{The model}

If the avian population is subject to Allee effect in system (1), then we have the following SI-SIR model:

$$
\left\{\begin{array}{l}
\frac{d S_{a}}{d t}=r_{a} S_{a}\left(1-\frac{S_{a}}{M_{a}}\right)\left(\frac{S_{a}}{m_{a}}-1\right)-\beta_{a} S_{a} I_{a} \\
\frac{d I_{a}}{d t}=\beta_{a} S_{a} I_{a}-\left(\mu_{a}+\delta_{a}\right) I_{a} \\
\frac{d S_{h}}{d t}=\Pi_{h}-\beta_{h} I_{a} S_{h}-\mu_{h} S_{h} \\
\frac{d I_{h}}{d t}=\beta_{h} I_{a} S_{h}-\left(\mu_{h}+\delta_{h}+\gamma\right) I_{h} \\
\frac{d R_{h}}{d t}=\gamma I_{h}-\mu_{h} R_{h}
\end{array}\right.
$$

where $r_{a}, M_{a}$ and $m_{a}\left(m_{a}<M_{a}\right)$ are the intrinsic growth rate, the maximal carrying capacity and the critical carrying capacity of the avian population, respectively, other assumptions and the meanings of other parameters remain unchanged. System (9) has a unique solution satisfying the initial conditions in $\mathbb{R}_{+}^{5}$ which is a positively invariant set.

Define the basic reproduction number by

$$
\mathcal{R}_{0,2}=\frac{\beta_{a}\left(M_{a}+m_{a}\right)\left(\mu_{a}+\delta_{a}\right)}{\left(\mu_{a}+\delta_{a}\right)^{2}+M_{a} m_{a} \beta_{a}^{2}} .
$$

We can deduce three disease-free equilibria given by $H_{1}\left(0,0, S_{h}^{*}, 0,0\right), H_{2}\left(m_{a}, 0, S_{h}^{*}, 0,0\right)$, and $H_{3}\left(M_{a}, 0, S_{h}^{*}, 0,0\right)$, where $S_{h}^{*}=\frac{\Pi_{h}}{\mu_{h}}$. If $\mathcal{R}_{0,2}>1$, we can also derive a unique endemic equilibrium given by $H_{4}\left(S_{a}^{* *}, I_{a}^{* *}, S_{h}^{* *}, I_{h}^{* *}, R_{h}^{* *}\right)$, where

$S_{a}^{* *}=\frac{\mu_{a}+\delta_{a}}{\beta_{a}}, I_{a}^{* *}=\frac{r_{a}}{\beta_{a}} \frac{\beta_{a}^{2} M_{a} m_{a}+\left(\mu_{a}+\delta_{a}\right)^{2}}{M_{a} m_{a} \beta_{a}^{2}}\left(\mathcal{R}_{0,2}-1\right), S_{h}^{* *}=\frac{\Pi_{h}}{\beta_{h} I_{a}^{* *}+\mu_{h}}, I_{h}^{* *}=\frac{\beta_{h} I_{a}^{* *} S_{h}^{* *}}{\mu_{h}+\delta_{h}+\gamma}, R_{h}^{* *}=\frac{\gamma I_{h}^{* *}}{\mu_{h}}$.

Comparing the relationship between $\mathcal{R}_{0,2}$ and 1 , we have the following results:

(i) $\mathcal{R}_{0,2}<1 \Leftrightarrow \frac{\mu_{a}+\delta_{a}}{\beta_{a}}<m_{a}$ or $\frac{\mu_{a}+\delta_{a}}{\beta_{a}}>M_{a}$;

(ii) $\mathcal{R}_{0,2}>1 \Leftrightarrow m_{a}<\frac{\mu_{a}+\delta_{a}}{\beta_{a}}<M_{a}$;

(iii) $\mathcal{R}_{0,2}=1 \Leftrightarrow \frac{\mu_{a}+\delta_{a}}{\beta_{a}}=m_{a}$ or $\frac{\mu_{a}+\delta_{a}}{\beta_{a}}=M_{a}$.

Before analyzing the dynamical behavior of the full model (9) with Allee effect, once again we first study the dynamical behavior of the avian-only subsystem.

\subsection{Analysis of the avian-only subsystem}

Consider the following avian-only subsystem:

$$
\left\{\begin{array}{l}
\frac{d S_{a}}{d t}=r_{a} S_{a}\left(1-\frac{S_{a}}{M_{a}}\right)\left(\frac{S_{a}}{m_{a}}-1\right)-\beta_{a} S_{a} I_{a} \\
\frac{d I_{a}}{d t}=\beta_{a} S_{a} I_{a}-\left(\mu_{a}+\delta_{a}\right) I_{a} .
\end{array}\right.
$$

Similarly, $\mathbb{R}_{+}^{2}$ is a positively invariant set of the subsystem (10). First we discuss its dynamical behavior in $\mathbb{R}_{+}^{2}$. 


\subsubsection{Local stability of the avian-only subsystem (10)}

The avian-only subsystem (10) always has three disease-free equilibria given by $O(0,0), A\left(m_{a}, 0\right)$ and $B\left(M_{a}, 0\right)$; if $\mathcal{R}_{0,2}>1$, then the subsystem also has a unique endemic equilibrium given by $E\left(S_{a}^{* *}, I_{a}^{* *}\right)$.

Lemma 3.1. (i) The disease-free equilibrium $O$ is always locally asymptotically stable but the disease-free equilibrium $A$ is always unstable; (ii) The disease-free equilibrium $B$ is unstable if $0<\frac{\mu_{a}+\delta_{a}}{\beta_{a}}<M_{a}$ but locally asymptotically stable if $\frac{\mu_{a}+\delta_{a}}{\beta_{a}} \geq M_{a}$ for positive trajectories; (iii) The endemic equilibrium $E$ is unstable if $m_{a}<\frac{\mu_{a}+\delta_{a}}{\beta_{a}}<\frac{M_{a}+m_{a}}{2}$ but locally asymptotically stable if $\frac{M_{a}+m_{a}}{2} \leq \frac{\mu_{a}+\delta_{a}}{\beta_{a}}<M_{a}$.

Proof. The characteristic equation of the Jacobian matrix of an arbitrary equilibrium $\left(S_{a}, I_{a}\right)$ is

$$
\left[\lambda-\left(r_{a}\left(\frac{-3 S_{a}^{2}+2\left(M_{a}+m_{a}\right) S_{a}}{M_{a} m_{a}}-1\right)-\beta_{a} I_{a}\right)\right]\left[\lambda-\left(\beta_{a} S_{a}-\mu_{a}-\delta_{a}\right)\right]+\beta_{a}^{2} S_{a} I_{a}=0 .
$$

(i) If $\left(S_{a}, I_{a}\right)=(0,0)$, the eigenvalues are $\lambda_{1}=-r_{a}<0, \lambda_{2}=-\left(\mu_{a}+\delta_{a}\right)<0$. Hence, the disease-free equilibrium $O$ is always locally asymptotically stable; If $\left(S_{a}, I_{a}\right)=\left(m_{a}, 0\right)$, the eigenvalues are $\lambda_{1}=\frac{\left(M_{a}-m_{a}\right) r_{a}}{M_{a}}>0, \lambda_{2}=\beta_{a}\left(m_{a}-\frac{\mu_{a}+\delta_{a}}{\beta_{a}}\right)$. Hence, the equilibrium $A$ is always unstable;

(ii) If $\left(S_{a}, I_{a}\right)=\left(M_{a}, 0\right)$, the eigenvalues are $\lambda_{1}=\frac{\left(m_{a}-M_{a}\right) r_{a}}{m_{a}}<0, \lambda_{2}=\beta_{a}\left(M_{a}-\frac{\mu_{a}+\delta_{a}}{\beta_{a}}\right)$. If $0<\frac{\mu_{a}+\delta_{a}}{\beta_{a}}<M_{a}$, then $\lambda_{2}>0$. Hence, the equilibrium $B$ is unstable; If $\frac{\mu_{a}+\delta_{a}}{\beta_{a}}>M_{a}$, then $\lambda_{2}<0$. Hence, the equilibrium $B$ is locally asymptotically stable;

(iii) If $m_{a}<\frac{\mu_{a}+\delta_{a}}{\beta_{a}}<M_{a}$ and $\left(S_{a}, I_{a}\right)=\left(S_{a}^{* *}, I_{a}^{* *}\right)$, the above characteristic equation becomes

$$
\lambda^{2}+a \lambda+b=0,
$$

where

$$
\begin{gathered}
a=-\frac{r_{a}}{M_{a} m_{a}} \frac{2\left(\mu_{a}+\delta_{a}\right)}{\beta_{a}}\left(\frac{M_{a}+m_{a}}{2}-\frac{\mu_{a}+\delta_{a}}{\beta_{a}}\right), \\
b=\frac{r_{a}\left(\mu_{a}+\delta_{a}\right)\left(\beta_{a}^{2} M_{a} m_{a}+\left(\mu_{a}+\delta_{a}\right)^{2}\right)}{M_{a} m_{a} \beta_{a}{ }^{2}}\left(\mathcal{R}_{0,2}-1\right) .
\end{gathered}
$$

Clearly, if $\frac{M_{a}+m_{a}}{2}<\frac{\mu_{a}+\delta_{a}}{\beta_{a}}<M_{a}$, then $a>0$ and $b>0$. Thus all eigenvalues have negative real parts and the endemic equilibrium $E$ is locally asymptotically stable; if $m_{a}<\frac{\mu_{a}+\delta_{a}}{\beta_{a}}<\frac{M_{a}+m_{a}}{2}$, then $a<0$ and $b>0$. Hence all the eigenvalues have positive real parts and the endemic equilibrium $E$ is unstable. If $\frac{\mu_{a}+\delta_{a}}{\beta_{a}}=\frac{M_{a}+m_{a}}{2}$, the characteristic equation (11) has purely imaginary eigenvalues $\pm i \omega$, where $\omega=\sqrt{\beta_{a}^{2} S_{a}^{* *} I_{a}^{* *}}>0$. In this case, the endemic equilibrium $E$ is a center or a fine focus.

Next, we shall study the type of the equilibrium $E$ if $\frac{\mu_{a}+\delta_{a}}{\beta_{a}}=\frac{M_{a}+m_{a}}{2}$. Making a transformation

$$
S=S_{a}-S_{a}^{* *}, \quad I=I_{a}-I_{a}^{* *}
$$

System (10) can be turned into

$$
\left\{\begin{array}{l}
\frac{d S}{d t}=r_{a}\left(S+S_{a}^{* *}\right)\left(1-\frac{S+S_{a}^{* *}}{M_{a}}\right)\left(\frac{S+S_{a}^{* *}}{m_{a}}-1\right)-\beta_{a}\left(S+S_{a}^{* *}\right)\left(I+I_{a}^{* *}\right) \\
\frac{d I}{d t}=\beta_{a}\left(S+S_{a}^{* *}\right)\left(I+I_{a}^{* *}\right)-\left(\mu_{a}+\delta_{a}\right)\left(I+I_{a}^{* *}\right) .
\end{array}\right.
$$

Simplifying system (12), it becomes

$$
\left\{\begin{array}{l}
\frac{d S}{d t}=-\beta_{a} S_{a}^{* *} I-\frac{r_{a} S_{a}^{* *}}{M_{a} m_{a}} S^{2}-\beta_{a} I S-\frac{r_{a}}{M_{a} m_{a}} S^{3} \\
\frac{d I}{d t}=\beta_{a} I_{a}^{* *} S+\beta_{a} I S
\end{array}\right.
$$


Let $x=S, y=\sqrt{\frac{S_{a}^{* *}}{I_{a}^{* *}}} I$. System $(13)$ can be written as

$$
\left\{\begin{array}{l}
\frac{d x}{d t}=-\sqrt{\beta_{a}^{2} S_{a}^{* *} I_{a}^{* *}} y-\frac{r_{a} S_{a}^{* *}}{M_{a} m_{a}} x^{2}-\frac{\beta_{a}}{\sqrt{\frac{S_{a}^{* *}}{I_{a}^{* *}}}} x y-\frac{r_{a}}{M_{a} m_{a}} x^{3} \\
\frac{d y}{d t}=\sqrt{\beta_{a}^{2} S_{a}^{* *} I_{a}^{* *}} x+\beta_{a} x y .
\end{array}\right.
$$

According to the Hopf bifurcation formula in Guckenheimer and Holmes [23] in two-dimensional systems

$$
\frac{d x}{d t}=-\omega y+f(x, y), \frac{d y}{d t}=\omega x+g(x, y)
$$

the singular point $(0,0)$ of system $(14)$ is a stable fine focus of order one for $c<0$, where

$$
c=\frac{f_{x x x}+f_{x y y}+g_{x x y}+g_{y y y}}{16}+\frac{f_{x y}\left(f_{x x}+f_{y y}\right)-g_{x y}\left(g_{x x}+g_{y y}\right)-f_{x x} g_{x x}+f_{y y} g_{y y}}{16 \omega},
$$

in which the partial derivatives are all evaluated at $(0,0)$ as follows: $f_{x x x}=\frac{-6 r_{a}}{M_{a} m_{a}}, f_{x y y}=0$, $g_{x x y}=0, g_{y y y}=0, f_{x x}=\frac{-2 r_{a} S_{a}^{* *}}{M_{a} m_{a}}, f_{x y}=\frac{-\beta_{a}}{\sqrt{\frac{S_{a}^{* *}}{I_{a}^{* *}}}}, f_{y y}=0, g_{x x}=0, g_{x y}=\beta_{a}, g_{y y}=0$. Then $c=\frac{-r_{a}}{4 M_{a} m_{a}}<0$. Thus, the trivial equilibrium $(0,0)$ of system (14) is a stable fine focus of order one. Hence, the endemic equilibrium $E$ of subsystem (10) is a stable fine focus of order one for $\frac{\mu_{a}+\delta_{a}}{\beta_{a}}=\frac{M_{a}+m_{a}}{2}$.

In summary, the endemic equilibrium $E$ of the avian-only subsystem (10) is locally asymptotically stable if $\frac{M_{a}+m_{a}}{2} \leq \frac{\mu_{a}+\delta_{a}}{\beta_{a}}<M_{a}$ and unstable if $m_{a}<\frac{\mu_{a}+\delta_{a}}{\beta_{a}}<\frac{M_{a}+m_{a}}{2}$.

Remark 3.2. If $\frac{\mu_{a}+\delta_{a}}{\beta_{a}}=m_{a}$, then the equilibrium $E$ coincides with the equilibrium $A$, which is a saddle-node and the disease-free equilibrium $A$ is unstable for positive trajectories; If $\frac{\mu_{a}+\delta_{a}}{\beta_{a}}=M_{a}$, then the equilibrium $E$ coincides with the equilibrium $B$, which is a saddle-node and the diseasefree equilibrium $B$ is locally asymptotically stable for positive trajectories.

In order to discuss the existence and uniqueness of limit cycles in the avian-only subsystem (10), we introduce a lemma.

Lemma 3.3. (Coppel $[15,16]$ ) Let $f(x)$ and $g(x)$ be continuously differentiable functions on an open interval $\left(r_{1}, r_{2}\right)$ and $\psi(y)$ be a continuously differentiable function on $\mathbb{R}$. Consider the Liénard system

$$
\left\{\begin{array}{l}
\frac{d x}{d t}=\psi(y)-\int_{x_{0}}^{x} f(u) d u \\
\frac{d y}{d t}=-g(x)
\end{array}\right.
$$

and assume that

(i) $\frac{d \psi(y)}{d y}>0$

(ii) there is a unique $x_{0} \in\left(r_{1}, r_{2}\right)$ such that $\left(x-x_{0}\right) g\left(x-x_{0}\right)>0$ for $x \neq x_{0}$ and $g\left(x_{0}\right)=0$;

(iii) $f\left(x_{0}\right) \frac{d}{d x}\left(\frac{f(x)}{g(x)}\right)<0$ for $x \neq x_{0}$.

Then system (15) has at most one limit cycle, and if it exists, it is hyperbolic.

Theorem 3.4. If $m_{a}<\frac{\mu_{a}+\delta_{a}}{\beta_{a}}<\frac{M_{a}+m_{a}}{2}$, then the avian-only subsystem (10) has a unique limit cycle which is hyperbolic. 
Proof. In order to apply Lemma 3.3, we make a transformation

$$
S_{a}=x, I_{a}=e^{y}, d t=-x^{-1} d \tau .
$$

System (10) can be written as

$$
\begin{cases}\frac{d x}{d \tau}=\beta_{a} e^{y}-r_{a}\left(1-\frac{x}{M_{a}}\right)\left(\frac{x}{m_{a}}-1\right) & \equiv \psi(y)-F(x) \\ \frac{d y}{d \tau}=\frac{\left(\mu_{a}+\delta_{a}\right)}{x}-\beta_{a} & \equiv-g(x)\end{cases}
$$

where $\psi(y)=\beta_{a} e^{y}, F(x)=r_{a}\left(1-\frac{x}{M_{a}}\right)\left(\frac{x}{m_{a}}-1\right)$, and $g(x)=\beta_{a}-\frac{\left(\mu_{a}+\delta_{a}\right)}{x}$.

Set $r_{1}=m_{a}, r_{2}=\frac{M_{a}+m_{a}}{2}$. We check the three conditions of Lemma 3.3:

(i) $\frac{d \psi(y)}{d y}=\beta_{a} e^{y}>0$.

(ii) $\frac{d g(x)}{d x}=\frac{\mu_{a}+\delta_{a}}{x^{2}}>0$. We choose $x_{0}=\frac{\mu_{a}+\delta_{a}}{\beta_{a}} \in\left(r_{1}, r_{2}\right)$, where $x_{0}$ satisfies that $g\left(x_{0}\right)=0$. Hence, $\left(x-x_{0}\right) g\left(x-x_{0}\right)>0$ for $x \neq x_{0}$.

(iii) $f(x)=\frac{d F(x)}{d x}=\frac{-2 r_{a}}{M_{a} m_{a}}\left(x-\frac{M_{a}+m_{a}}{2}\right), f\left(x_{0}\right)=\frac{-2 r_{a}}{M_{a} m_{a}}\left(x_{0}-\frac{M_{a}+m_{a}}{2}\right)>0, \frac{f(x)}{g(x)}=\frac{-r_{a} x\left(2 x-\left(M_{a}+m_{a}\right)\right)}{M_{a} m_{a}\left(\beta_{a} x-\left(\mu_{a}+\delta_{a}\right)\right)}$, $\frac{d}{d x}\left(\frac{f(x)}{g(x)}\right)=\frac{h(x)}{M_{a} m_{a}\left(\beta_{a} x-\left(\mu_{a}+\delta_{a}\right)\right)^{2}}$, where $h(x)=r_{a}\left(-2 \beta_{a} x^{2}+4\left(\mu_{a}+\delta_{a}\right) x-\left(\mu_{a}+\delta_{a}\right)\left(M_{a}+m_{a}\right)\right)$, $\frac{d}{d x}(h(x))=-4 \beta_{a} r_{a}\left(x-\frac{\mu_{a}+\delta_{a}}{\beta_{a}}\right)$. When $\widetilde{x}=\frac{\mu_{a}+\delta_{a}}{\beta_{a}}=x_{0}, h^{\prime}(\widetilde{x})=0, h^{\prime}(x)>0$ for $m_{a}<x<\widetilde{x}$ and $h^{\prime}(x)<0$ for $\widetilde{x}<x<\frac{m_{a}+M_{a}}{2}$. $h(\widetilde{x})=2\left(\mu_{a}+\delta_{a}\right) r_{a}\left(\frac{\mu_{a}+\delta_{a}}{\beta_{a}}-\frac{M_{a}+m_{a}}{2}\right)<0$. Hence, we have $h(x)<h(\widetilde{x})<0, f\left(x_{0}\right) \frac{d}{d x}\left(\frac{f(x)}{g(x)}\right)<0$ for $m_{a}<x<\frac{M_{a}+m_{a}}{2}$ and $x \neq x_{0}$.

Thus, system (16) satisfies the three conditions of Lemma 3.3. So the avian-only subsystem (10) has at most one limit cycle, and it is hyperbolic.

Next, we prove the existence of a limit cycle of subsystem (10). We choose $\beta_{a}$ as a perturbed parameter. The equation $\frac{\mu_{a}+\delta_{a}}{\beta_{a}}=\frac{M_{a}+m_{a}}{2}$ implies that $\beta_{a}=\frac{2\left(\mu_{a}+\delta_{a}\right)}{M_{a}+m_{a}}$. Set $\mu=\beta_{a}-\frac{2\left(\mu_{a}+\delta_{a}\right)}{M_{a}+m_{a}}$, where $|\mu| \ll 1$. According to Lemma 3.1, we have the following results:

If $\mu<0$ (i.e., $\frac{\mu_{a}+\delta_{a}}{\beta_{a}}>\frac{M_{a}+m_{a}}{2}$ ), then the endemic equilibrium $E$ is locally asymptotically stable; If $\mu=0$ (i.e., $\frac{\mu_{a}+\delta_{a}}{\beta_{a}}=\frac{M_{a}+m_{a}}{2}$ ), then the endemic equilibrium $E$ is a stable fine focus of order one; If $\mu>0$ (i.e., $\frac{\mu_{a}+\delta_{a}}{\beta_{a}}<\frac{M_{a}+m_{a}}{2}$ ), then the endemic equilibrium $E$ is an unstable focus.

By the results in Zhang and Feng [60] (p.207), there exists at least one stable limit cycle in the neighborhood of the endemic equilibrium $E$ of system (10) for sufficient small $\mu>0$. Thus, system (10) has a unique limit cycle which is hyperbolic for $m_{a}<\frac{\mu_{a}+\delta_{a}}{\beta_{a}}<\frac{M_{a}+m_{a}}{2}$.

\subsubsection{Global stability of the avian-only subsystem (10)}

In order to study global stability of these equilibria, we need to analyze the critical point at infinity of the avian-only subsystem (10).

Making a Poincaré transformation

$$
S_{a}=\frac{1}{z}, I_{a}=\frac{u}{z} \text { or } z=\frac{1}{S_{a}}, u=\frac{I_{a}}{S_{a}}
$$

and let $d \tau=\frac{d t}{z^{2}}$. Then system (10) can be written as

$$
\left\{\begin{array}{l}
\frac{d u}{d \tau}=\frac{r_{a}}{M_{a} m_{a}} u+\left(\beta_{a}-\frac{r_{a}\left(M_{a}+m_{a}\right)}{M_{a} m_{a}}\right) u z+\beta_{a} u^{2} z+\left(r_{a}-\mu_{a}-\delta_{a}\right) u z^{2} \\
\frac{d z}{d \tau}=\frac{r_{a}}{M_{a} m_{a}} z-\frac{r_{a}\left(M_{a}+m_{a}\right)}{M_{a} m_{a}} z^{2}+\beta_{a} u z^{2}+r_{a} z^{3} .
\end{array}\right.
$$

It is clear to see that there is a unique equilibrium $C(0,0)$ on the $u$-axis. The eigenvalues of the Jacobian matrix of the equilibrium $C(0,0)$ of system (17) are $\lambda_{1}=\lambda_{2}=\frac{r_{a}}{M_{a} m_{a}}$. Hence, the equilibrium $C(0,0)$ is an unstable node. 
Making another Poincaré transformation

$$
S_{a}=\frac{v}{z}, I_{a}=\frac{1}{z} \text { or } z=\frac{1}{I_{a}}, v=\frac{S_{a}}{I_{a}}
$$

and letting $d \tau=\frac{d t}{z^{2}}$. Then system (10) is transformed into

$$
\left\{\begin{array}{l}
\frac{d v}{d \tau}=-\beta_{a} v z+\left(\mu_{a}+\delta_{a}\right) v z^{2}-\beta_{a} v^{2} z+r_{a} v\left(z-\frac{v}{M_{a}}\right)\left(\frac{v}{m_{a}}-z\right) \\
\frac{d z}{d \tau}=-\beta_{a} v z^{2}+\left(\mu_{a}+\delta_{a}\right) z^{3}
\end{array}\right.
$$

Let $z=0$. Then system (18) has an equilibrium $D(0,0)$ which is a higher order singular point. The geometric property of the higher order singular point $D(0,0)$ of system $(18)$ is decided by the following system:

$$
\left\{\begin{array}{l}
\frac{d v}{d \tau}=-\beta_{a} v z \\
\frac{d z}{d \tau}=-\beta_{a} v z^{2}+\left(\mu_{a}+\delta_{a}\right) z^{3} .
\end{array}\right.
$$

Making a time transformation

$$
d \tau=d \tau_{1} / z
$$

Then system (19) becomes

$$
\left\{\begin{array}{l}
\frac{d v}{d \tau_{1}}=-\beta_{a} v \\
\frac{d z}{d \tau_{1}}=-\beta_{a} v z+\left(\mu_{a}+\delta_{a}\right) z^{2}
\end{array}\right.
$$

System (20) has a unique equilibrium $(0,0)$ which is a higher order singular point with one of the eigenvalues being zero. By the results in Zhang et al. [61], the equilibrium $(0,0)$ is a saddle-node.

Thus, we have the following results: system (10) has two critical points at infinity given by $C(0,0)$ and $D(0,0)$, where $C(0,0)$ corresponds with the critical point at infinity of the $S_{a}$-axis and is an unstable node, and $D(0,0)$ corresponds with the critical point at infinity of the $I_{a}$-axis and is a saddle-node.

Hence, we can always divide the region $\mathbb{R}_{+}^{2}$ into sub-regions $D_{1}$ and $D_{2}$ as follows:

(i) If $0<\frac{\mu_{a}+\delta_{a}}{\beta_{a}} \leq m_{a}$, then the sub-region $D_{1}$ is surrounded by the saddle-node separatrix $B D$, curve $D O$, and curve $O B$; the sub-region $D_{2}$ is surrounded by the saddle-node separatrix $B D$, curve $C D$, and curve $C B$.

(ii) If $m_{a}<\frac{\mu_{a}+\delta_{a}}{\beta_{a}}<\frac{m_{a}+M_{a}}{2}$, then the sub-region $D_{1}$ is surrounded by the saddle-node separatrix $D A$, curve $D O$, and curve $A O$; the sub-region $D_{2}$ is surrounded by the saddle-node separatrix $D A$, curve $A C$, and curve $C D$.

(iii) If $\frac{m_{a}+M_{a}}{2} \leq \frac{\mu_{a}+\delta_{a}}{\beta_{a}}<M_{a}$, then the sub-region $D_{1}$ is surrounded by the saddle-node separatrix $D A$, curve $D O$, and curve $A O$; the sub-region $D_{2}$ is surrounded by the saddle-node separatrix $D A$, curve $A C$, and curve $C D$.

(iv) If $\frac{\mu_{a}+\delta_{a}}{\beta_{a}} \geq M_{a}$, then the sub-region $D_{1}$ is surrounded by the saddle-node separatrix $D A$, curve $D O$, and curve $A O$; the sub-region $D_{2}$ is surrounded by the saddle-node separatrix $D A$, curve $A C$, and curve $C D$.

The global dynamics of the avian-only subsystem (10) can be summarized in the following theorem.

Theorem 3.5. (i) The disease-free equilibrium $O$ of the avian-only subsystem (10) is always globally asymptotically stable in $D_{1}$; (ii) If $m_{a}<\frac{\mu_{a}+\delta_{a}}{\beta_{a}}<\frac{m_{a}+M_{a}}{2}$, then there is a limit cycle in the neighborhood of the endemic equilibrium $E$ of the avian-only subsystem (10) which is globally 
asymptotically stable in $D_{2}$; (iii) If $\frac{m_{a}+M_{a}}{2} \leq \frac{\mu_{a}+\delta_{a}}{\beta_{a}}<M_{a}$, then the endemic equilibrium $E$ of the avian-only subsystem (10) is globally asymptotically stable in $D_{2}$; (iv) If $\frac{\mu_{a}+\delta_{a}}{\beta_{a}} \geq M_{a}$, then the disease-free equilibrium $B$ of the avian-only subsystem (10) is globally asymptotically stable in $D_{2}$.

Proof. Lemma 3.1 implies that the disease-free equilibrium $O$ is always locally asymptotically stable, the endemic equilibrium $E$ is locally asymptotically stable for $\frac{m_{a}+M_{a}}{2} \leq \frac{\mu_{a}+\delta_{a}}{\beta_{a}}<M_{a}$, and the disease-free equilibrium $B$ is locally asymptotically stable for $\frac{\mu_{a}+\delta_{a}}{\beta_{a}} \geq M_{a}$.

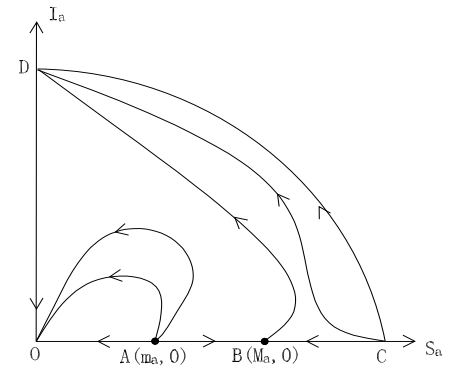

(a) $0<\frac{\mu_{a}+\delta_{a}}{\beta_{a}} \leq m_{a}$

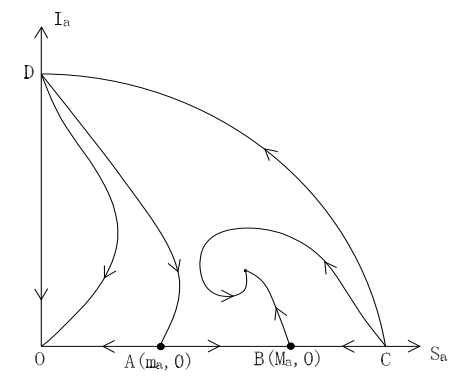

(c) $\frac{m_{a}+M_{a}}{2} \leq \frac{\mu_{a}+\delta_{a}}{\beta_{a}}<M_{a}$

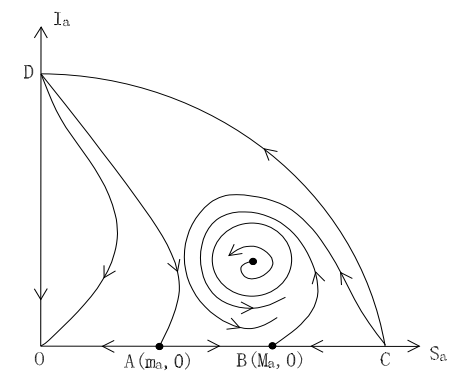

(b) $m_{a}<\frac{\mu_{a}+\delta_{a}}{\beta_{a}}<\frac{m_{a}+M_{a}}{2}$

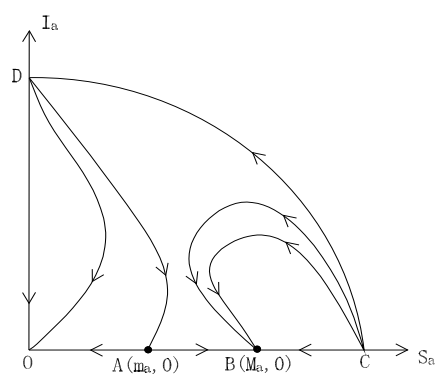

(d) $\frac{\mu_{a}+\delta_{a}}{\beta_{a}} \geq M_{a}$

Figure 1: The plots are the global phase portraits of the avian-only subsystem (10) with respect to $\frac{\mu_{a}+\delta_{a}}{\beta_{a}}$. (a) $0<\frac{\mu_{a}+\delta_{a}}{\beta_{a}} \leq m_{a}$; (b) $m_{a}<\frac{\mu_{a}+\delta_{a}}{\beta_{a}}<\frac{m_{a}+M_{a}}{2}$; (c) $\frac{m_{a}+M_{a}}{2} \leq \frac{\mu_{a}+\delta_{a}}{\beta_{a}}<M_{a}$; (d) $\frac{\mu_{a}+\delta_{a}}{\beta_{a}} \geq M_{a}$.

(i) If $\left(S_{a}, I_{a}\right) \in D_{1}$, it should be noted that subsystem (10) has no endemic equilibrium in the interior of $D_{1}$ and the $S_{a^{-}}$and $I_{a^{-}}$axes are positively invariant, so there is no limit cycle in $D_{1}$. Hence, the disease-free equilibrium $O$ is globally asymptotically stable in $D_{1}$ (see Fig. 1(a)).

(ii) If $m_{a}<\frac{\mu_{a}+\delta_{a}}{\beta_{a}}<\frac{M_{a}+m_{a}}{2}$ and $\left(S_{a}, I_{a}\right) \in D_{2}$, by Lemma 3.1 and Theorem 3.4, subsystem (10) has a unique limit cycle which is hyperbolic in $D_{2}$ and the endemic equilibrium $E$ is an unstable focus, thus we can deduce that the limit cycle is internally stable (semistable from inside); according to Lemma 3.1, the equilibrium $B$ is an unstable node, the infinite point $C$ is an unstable node, the infinite point $D$ is a saddle-node, and the saddle-node separatrix $D A$ is a curve from the point $D$ to the point $A$, thus solutions starting from the exterior of the limit cycle are tending to the limit cycle, that is, the limit cycle is externally stable (semistable from outside). Hence, the limit cycle is globally asymptotically stable in $D_{2}$ (see Fig. 1(b)).

(iii) If $\frac{m_{a}+M_{a}}{2} \leq \frac{\mu_{a}+\delta_{a}}{\beta_{a}}<M_{a}$ and $\left(S_{a}, I_{a}\right) \in D_{2}$, by Theorem 3.4, we know that there is no limit cycle in the neighborhood of the endemic equilibrium $E$ in the $D_{2}$. On the other hand, according to Lemma 3.1, the endemic equilibrium $E$ is a stable focus, the equilibrium $B$ is an unstable saddle, the infinite point $C$ is an unstable node, the infinite point $D$ is a saddle-node, and the saddle-node separatrix $D A$ is a curve from the point $D$ to the point $A$, thus solutions starting from the region $D_{2}$ are tending to the equilibrium $E$. Hence, the endemic equilibrium $E$ is globally asymptotically stable in $D_{2}$ (see Fig. 1(c)).

(iv) If $\frac{\mu_{a}+\delta_{a}}{\beta_{a}} \geq M_{a}$ and $\left(S_{a}, I_{a}\right) \in D_{2}$, it should be noted that there is no endemic equilibrium in the region $D_{2}$, so there is no limit cycle. By Lemma 3.1, the equilibrium $B$ is a stable node, 
the infinite point $C$ is an unstable node, the infinite point $D$ is a saddle-node, and the saddle-node separatrix $D A$ is a curve from the point $D$ to the point $A$, thus all solutions starting from the region $D_{2}$ are tending to the disease-free equilibrium $B$. Hence, the disease-free equilibrium $B$ is globally asymptotically stable in $D_{2}$ (see Fig. 1(d)).

\subsection{Analysis of the full system}

Since the first four equations of system (9) are independent of the variable $R_{h}$, similarly we only analyze the dynamical behavior of the following equivalent system

$$
\left\{\begin{array}{l}
\frac{d S_{a}}{d t}=r_{a} S_{a}\left(1-\frac{S_{a}}{M_{a}}\right)\left(\frac{S_{a}}{m_{a}}-1\right)-\beta_{a} S_{a} I_{a} \\
\frac{d I_{a}}{d t}=\beta_{a} S_{a} I_{a}-\left(\mu_{a}+\delta_{a}\right) I_{a} \\
\frac{d S_{h}}{d t}=\Pi_{h}-\beta_{h} I_{a} S_{h}-\mu_{h} S_{h} \\
\frac{d I_{h}}{d t}=\beta_{h} I_{a} S_{h}-\left(\mu_{h}+\delta_{h}+\gamma\right) I_{h}
\end{array}\right.
$$

We discuss the dynamical behavior of system (21) in its positively invariant set $\mathbb{R}_{+}^{4}$.

\subsubsection{Local stability of the full system (21)}

System (21) has three equilibria given by $O_{a h}\left(0,0, S_{h}^{*}, 0\right), A_{a h}\left(m_{a}, 0, S_{h}^{*}, 0\right)$, and $B_{a h}\left(M_{a}, 0, S_{h}^{*}, 0\right)$. If $\mathcal{R}_{0,2}>1$, system (21) also has a unique endemic equilibrium given by $E_{a h}\left(S_{a}^{* *}, I_{a}^{* *}, S_{h}^{* *}, I_{h}^{* *}\right)$.

Lemma 3.6. (i) The disease-free equilibrium $O_{a h}$ is always locally asymptotically stable and the disease-free equilibrium $A_{a h}$ is always unstable; (ii) If $\frac{\mu_{a}+\delta_{a}}{\beta_{a}}<M_{a}$, then the disease-free equilibrium $B_{a h}$ is unstable; if $\frac{\mu_{a}+\delta_{a}}{\beta_{a}} \geq M_{a}$, then the disease-free equilibrium $B_{a h}$ is locally asymptotically stable; (iii) If $m_{a}<\frac{\mu_{a}+\delta_{a}}{\beta_{a}}<\frac{M_{a}+m_{a}}{2}$, then the endemic equilibrium $E_{a h}$ is unstable; if $\frac{M_{a}+m_{a}}{2} \leq$ $\frac{\mu_{a}+\delta_{a}}{\beta_{a}}<M_{a}$, then the endemic equilibrium $E_{a h}$ is locally asymptotically stable.

Proof. The characteristic equation of the Jacobian matrix of an arbitrary equilibrium $\left(S_{a}, I_{a}, S_{h}, I_{h}\right)$ of system (21) is given by

$$
\begin{aligned}
& {\left[\left(\lambda-\frac{r_{a}\left(-3 S_{a}^{2}+2\left(M_{a}+m_{a}\right) S_{a}-M_{a} m_{a}\right)}{M_{a} m_{a}}-\beta_{a} I_{a}\right)\left(\lambda-\beta_{a} S_{a}+\mu_{a}+\delta_{a}\right)+\beta_{a}^{2} S_{a} I_{a}\right]} \\
& \quad\left(\lambda+\beta_{h} I_{a}+\mu_{h}\right)\left(\lambda+\mu_{h}+\delta_{h}+\gamma\right)=0 .
\end{aligned}
$$

(i) If $\left(S_{a}, I_{a}, S_{h}, I_{h}\right)=\left(0,0, S_{h}^{*}, 0\right)$, the eigenvalues are $\lambda_{1}=-r_{a}, \lambda_{2}=-\left(\mu_{a}+\delta_{a}\right), \lambda_{3}=-\mu_{h}$, $\lambda_{4}=-\left(\mu_{h}+\delta_{h}+\gamma\right)$. Obviously, these eigenvalues are negative. Hence, the disease-free equilibrium $O_{a h}$ is always locally asymptotically stable; If $\left(S_{a}, I_{a}, S_{h}, I_{h}\right)=\left(m_{a}, 0, S_{h}^{*}, 0\right)$, the eigenvalues are

$$
\lambda_{1}=\frac{\left(M_{a}-m_{a}\right) r_{a}}{M_{a}}>0, \lambda_{2}=\beta_{a}\left(m_{a}-\frac{\mu_{a}+\delta_{a}}{\beta_{a}}\right), \lambda_{3}=-\mu_{h}, \lambda_{4}=-\left(\mu_{h}+\delta_{h}+\gamma\right) .
$$

Since one of the eigenvalues is positive, the disease-free equilibrium $A_{a h}$ is always unstable.

(ii) If $\left(S_{a}, I_{a}, S_{h}, I_{h}\right)=\left(M_{a}, 0, S_{h}^{*}, 0\right)$, the eigenvalues are

$$
\lambda_{1}=\frac{\left(m_{a}-M_{a}\right) r_{a}}{m_{a}}, \lambda_{2}=\beta_{a}\left(M_{a}-\frac{\mu_{a}+\delta_{a}}{\beta_{a}}\right), \lambda_{3}=-\mu_{h}, \lambda_{4}=-\left(\mu_{h}+\delta_{h}+\gamma\right) .
$$

Obviously, if $\frac{\mu_{a}+\delta_{a}}{\beta_{a}}>M_{a}$, all the above eigenvalues are negative, the disease-free equilibrium $B_{a h}$ is locally asymptotically stable; If $0<\frac{\mu_{a}+\delta_{a}}{\beta_{a}}<M_{a}$, then $\lambda_{2}>0$. Hence the disease-free equilibrium $B_{a h}$ is unstable. 
(iii) If $m_{a}<\frac{\mu_{a}+\delta_{a}}{\beta_{a}}<M_{a}$ and $\left(S_{a}, I_{a}, S_{h}, I_{h}\right)=\left(S_{a}^{* *}, I_{a}^{* *}, S_{h}^{* *}, I_{h}^{* *}\right)$, the above characteristic equation becomes

$$
\left(\lambda+\mu_{h}+\delta_{h}+\gamma\right)\left(\lambda+\beta_{h} I_{a}^{* *}+\mu_{h}\right)\left(\lambda^{2}+a \lambda+b\right)=0,
$$

where the meanings of $a$ and $b$ are the same as in the characteristic equation (11). Since the characteristic equation (22) has at least two negative eigenvalues $\lambda=-\left(\mu_{h}+\delta_{h}+\gamma\right), \lambda=-\left(\beta_{h} I_{a}^{* *}+\right.$ $\left.\mu_{h}\right)$, the local stability of the endemic equilibrium $E_{a h}$ of system (21) is decided by the equation $\lambda^{2}+a \lambda+b=0$. Lemma 3.1 implies that if $\frac{M_{a}+m_{a}}{2} \leq \frac{\mu_{a}+\delta_{a}}{\beta_{a}}<M_{a}$, then the endemic equilibrium $E_{a h}$ is locally asymptotically stable; if $m_{a}<\frac{\mu_{a}+\delta_{a}}{\beta_{a}}<\frac{M_{a}+m_{a}}{2}$, then the endemic equilibrium $E_{a h}$ is unstable.

Remark 3.7. If $\frac{\mu_{a}+\delta_{a}}{\beta_{a}}=M_{a}$, then the equilibrium $E_{a h}$ coincides with the equilibrium $B_{a h}$, which is a saddle-node and is locally asymptotically stable for positive trajectories.

\subsubsection{Global stability of the full system (21)}

Set $E_{1}=\left\{\left(S_{a}, I_{a}, S_{h}, I_{h}\right):\left(S_{a}, I_{a}\right) \in D_{1}, S_{h} \geq 0, I_{h} \geq 0\right\}$ and $E_{2}=\left\{\left(S_{a}, I_{a}, S_{h}, I_{h}\right):\left(S_{a}, I_{a}\right) \in\right.$ $\left.D_{2}, S_{h} \geq 0, I_{h} \geq 0\right\}$, where $D_{1}$ and $D_{2}$ are defined in Theorem 3.5.

Theorem 3.8. (i) The disease-free equilibrium $O_{a h}$ of system (21) is always globally asymptotically stable in $E_{1}$; (ii) If $\frac{M_{a}+m_{a}}{2} \leq \frac{\mu_{a}+\delta_{a}}{\beta_{a}}<M_{a}$, only the endemic equilibrium $E_{a h}\left(S_{a}^{* *}, I_{a}^{* *}, S_{h}^{* *}, I_{h}^{* *}\right)$ of system (21) is globally asymptotically stable in $E_{2}$; (iii) If $\frac{\mu_{a}+\delta_{a}}{\beta_{a}} \geq M_{a}$, only the disease-free equilibrium $B_{a h}\left(M_{a}, 0, S_{h}^{*}, 0\right)$ of system (21) is globally asymptotically stable in the region $E_{2}$.

Proof. (i) If $\left(S_{a}, I_{a}, S_{h}, I_{h}\right) \in E_{1}$, then $\left(S_{a}, I_{a}\right) \in D_{1}$. According to Theorem 3.5, the diseasefree equilibrium $O$ of the avian-only subsystem (10) is always globally asymptotically stable in the region $D_{1}$. To prove the global stability of the disease-free equilibrium $O_{a h}$, we only need to consider the system (21) with the avian components already at the disease-free steady state, given by

$$
\left\{\begin{array}{l}
\frac{d S_{h}}{d t}=\Pi_{h}-\mu_{h} S_{h} \\
\frac{d I_{h}}{d t}=-\left(\mu_{h}+\delta_{h}+\gamma\right) I_{h} .
\end{array}\right.
$$

Obviously, $I_{h} \rightarrow 0, S_{h} \rightarrow S_{h}^{*}$ if $t \rightarrow \infty$. Hence, the equilibrium $O_{a h}$ is always globally asymptotically stable in the region $E_{1}$.

(ii) If $\frac{M_{a}+m_{a}}{2} \leq \frac{\mu_{a}+\delta_{a}}{\beta_{a}}<M_{a}$ and $\left(S_{a}, I_{a}, S_{h}, I_{h}\right) \in E_{2}$, then $\left(S_{a}, I_{a}\right) \in D_{2}$. According to Theorem 3.5, the endemic equilibrium $E$ of the subsystem (10) is globally asymptotically stable in the region $D_{2}$. To prove the global stability of the endemic equilibrium $E_{a h}$, we consider system (21) with the avian components already at the endemic steady state given by

$$
\left\{\begin{array}{l}
\frac{d S_{h}}{d t}=\Pi_{h}-\beta_{a} I_{a}^{* *} S_{h}-\mu_{h} S_{h} \\
\frac{d I_{h}}{d t}=\beta_{a} I_{a}^{* *} S_{h}-\left(\mu_{h}+\delta_{h}+\gamma\right) I_{h} .
\end{array}\right.
$$

According to the proof of Theorem 2.6(ii), $S_{h} \rightarrow S_{h}^{* *}, I_{h} \rightarrow I_{h}^{* *}$ if $t \rightarrow \infty$. Hence, the endemic equilibrium $E_{a h}$ is globally asymptotically stable in the region $E_{2}$.

(iii) If $\frac{\mu_{a}+\delta_{a}}{\beta_{a}} \geq M_{a}$ and $\left(S_{a}, I_{a}, S_{h}, I_{h}\right) \in E_{2}$, then $\left(S_{a}, I_{a}\right) \in D_{2}$. By Theorem 3.5 , the diseasefree equilibrium $B$ of the subsystem (10) is globally asymptotically stable in the region $D_{2}$. To prove the global stability of the disease-free equilibrium $B_{a h}$, we consider system (21) with the avian components already at the disease-free steady state, given by

$$
\left\{\begin{array}{l}
\frac{d S_{h}}{d t}=\Pi_{h}-\mu_{h} S_{h} \\
\frac{d I_{h}}{d t}=-\left(\mu_{h}+\delta_{h}+\gamma\right) I_{h} .
\end{array}\right.
$$


Obviously, $I_{h} \rightarrow 0, S_{h} \rightarrow S_{h}^{*}$ if $t \rightarrow \infty$. Hence, the disease-free equilibrium $B_{a h}$ is globally asymptotically stable in the region $E_{2}$.

Lemma 3.9. The full system (21) has a unique periodic solution if and only if the subsystem (10) has a unique limit cycle.

Proof. At first, we prove the sufficient condition. According to Theorem 3.4, the subsystem (10) has a unique limit cycle. Let the $\omega$-periodic solution $\left(\tilde{S}_{a}(t), \tilde{I}_{a}(t)\right)$ be the unique limit cycle of the subsystem (10). We will prove that the third equation of system (21) has a unique $\omega$-periodic solution $\tilde{S}_{h}(t)$.

Any solution of the third equation of system (21) can be represented by

$$
S_{h}(t)=e^{-\beta_{h} \int_{t_{0}}^{t} I_{a}(s) d s-\mu_{h} t}\left[e^{\mu_{h} t_{0}} S_{h}\left(t_{0}\right)+\Pi_{h} \int_{t_{0}}^{t} e^{\beta_{h} \int_{t_{0}}^{s} I_{a}(u) d u} e^{\mu_{h} s} d s\right]
$$

where $S_{h}\left(t_{0}\right)$ is the initial value of $S_{h}(t)$. Thus, all solutions of the third equation of system (21) on the three-dimensional cylinder

$$
\Gamma \times \mathbb{R}^{+} \times \mathbb{R}^{+}=\Gamma \times[0, \infty) \times[0, \infty)
$$

are denoted as

$$
S_{h}(t)=e^{-\beta_{h} \int_{t_{0}}^{t} \tilde{I}_{a}(s) d s-\mu_{h} t}\left[e^{\mu_{h} t_{0}} S_{h}\left(t_{0}\right)+\Pi_{h} \int_{t_{0}}^{t} e_{h}^{\beta} \int_{t_{0}}^{s} \tilde{I}_{a}(u) d u e^{\mu_{h} s} d s\right]
$$

where $\Gamma=\left\{\left(\tilde{S}_{a}(t), \tilde{I}_{a}(t)\right): t \in[0, \omega]\right\}, R^{+}=[0, \infty)$.

In (25), we have

$$
\begin{aligned}
& S_{h}(t+\omega)= e^{-\beta_{h} \int_{t_{0}}^{t+\omega} \tilde{I}_{a}(s) d s-\mu_{h}(t+\omega)}\left[e^{\mu_{h} t_{0}} S_{h}\left(t_{0}\right)+\Pi_{h} \int_{t_{0}}^{t+\omega} e^{\beta_{h} \int_{t_{0}}^{s} \tilde{I}_{a}(u) d u} e^{\mu_{h} s} d s\right] \\
&= e^{-\beta_{h} \int_{t_{0}}^{t_{0}+\omega} \tilde{I}_{a}(s) d s} e^{-\beta_{h} \int_{t_{0}+\omega}^{t+\omega} \tilde{I}_{a}(s) d s} e^{-\mu_{h}(t+\omega)}\left[e^{\mu_{h} t_{0}} S_{h}\left(t_{0}\right)\right. \\
&\left.\quad+\Pi_{h} \int_{t_{0}}^{t_{0}+\omega} e^{\beta_{h} \int_{t_{0}}^{s} \tilde{I}_{a}(u) d u} e^{\mu_{h} s} d s+\Pi_{h} \int_{t_{0}+\omega}^{t+\omega} e^{\beta_{h} \int_{t_{0}}^{s} \tilde{I}_{a}(u) d u} e^{\mu_{h} s} d s\right] \\
&= e^{-\beta_{h} \int_{t_{0}+\omega}^{t+\omega} \tilde{I}_{a}(s) d s} e^{-\mu_{h} t}\left\{e ^ { - \beta _ { h } \int _ { t _ { 0 } } ^ { t _ { 0 } + \omega } \tilde { I } _ { a } ( s ) d s } e ^ { - \mu _ { h } \omega } \left[e^{\mu_{h} t_{0}} S_{h}\left(t_{0}\right)\right.\right. \\
&\left.\left.\quad+\Pi_{h} \int_{t_{0}}^{t_{0}+\omega} e^{\beta_{h} \int_{t_{0}}^{s} \tilde{I}_{a}(u) d u} e^{\mu_{h} s} d s\right]+\Pi_{h} \int_{t_{0}+\omega}^{t+\omega} e^{\beta_{h}\left(-\int_{t_{0}}^{t_{0}+\omega}+\int_{t_{0}}^{s}\right) \tilde{I}_{a}(u) d u} e^{\mu_{h}(s-\omega)} d s\right\} \\
&=e^{-\beta_{h} \int_{t_{0}}^{t} \tilde{I}_{a}(s) d s} e^{-\mu_{h} t}\left\{e ^ { - \beta _ { h } \int _ { 0 } ^ { \omega } \tilde { I } _ { a } ( s ) d s } e ^ { - \mu _ { h } \omega } \left[e^{\mu_{h} t_{0}} S_{h}\left(t_{0}\right)\right.\right. \\
&\left.\left.\quad+\Pi_{h} \int_{t_{0}}^{t_{0}+\omega} e^{\beta_{h} \int_{t_{0}}^{s} \tilde{I}_{a}(u) d u} e^{\mu_{h} s} d s\right]+\Pi_{h} \int_{t_{0}}^{t} e^{\beta_{h} \int_{t_{0}}^{s} \tilde{I}_{a}(u) d u} e^{\mu_{h} s} d s\right\} .
\end{aligned}
$$

If

$$
e^{-\beta_{h} \int_{0}^{\omega} \tilde{I}_{a}(s) d s} e^{-\mu_{h} \omega}\left[e^{\mu_{h} t_{0}} S_{h}\left(t_{0}\right)+\Pi_{h} \int_{t_{0}}^{t_{0}+\omega} e^{\beta_{h} \int_{t_{0}}^{s} \tilde{I}_{a}(u) d u} e^{\mu_{h} s} d s\right]=e^{\mu_{h} t_{0}} S_{h}\left(t_{0}\right),
$$

i.e., if

$$
S_{h}^{*}\left(t_{0}\right)=\frac{\Pi_{h} \int_{t_{0}}^{t_{0}+\omega} e^{\beta_{h} \int_{t_{0}}^{s} \tilde{I}_{a}(u) d u} e^{\mu_{h} s} d s}{e^{\mu_{h} t_{0}}\left[e^{\beta_{h} \int_{0}^{\omega} \tilde{I}_{a}(s) d s} e^{\mu_{h} \omega}-1\right]},
$$

then

$$
\tilde{S}_{h}(t)=e^{-\beta_{h} \int_{t_{0}}^{t} \tilde{I}_{a}(s) d s-\mu_{h} t}\left[e^{\mu_{h} t_{0}} S_{h}^{*}\left(t_{0}\right)+\Pi_{h} \int_{t_{0}}^{t} e^{\beta_{h} \int_{t_{0}}^{s} \tilde{I}_{a}(u) d u} e^{\mu_{h} s} d s\right]
$$

is a unique periodic solution of the third equation of system (21). 
Similarly,

$$
\tilde{I}_{h}(t)=e^{-\left(\mu_{h}+\delta_{h}+\gamma\right) t}\left[e^{\left(\mu_{h}+\delta_{h}+\gamma\right) t_{0}} I_{h}^{*}\left(t_{0}\right)+\beta_{h} \int_{t_{0}}^{t} e^{\left(\mu_{h}+\delta_{h}+\gamma\right) s} \tilde{I}_{a}(s) \tilde{S}_{h}(s) d s\right]
$$

is a unique periodic solution of the fourth equation of system (21), where

$$
I_{h}^{*}\left(t_{0}\right)=\frac{\beta_{h} \int_{t_{0}}^{t_{0}+\omega} e^{\left(\mu_{h}+\delta_{h}+\gamma\right) s} \tilde{I}_{a}(s) \tilde{S}_{h}(s) d s}{e^{\left(\mu_{h}+\delta_{h}+\gamma\right) t_{0}}\left[e^{\left(\mu_{h}+\delta_{h}+\gamma\right) \omega}-1\right]} .
$$

Hence, $\left(\tilde{S}_{a}(t), \tilde{I}_{a}(t), \tilde{S}_{h}(t), \tilde{I}_{h}(t)\right)$ is a unique periodic solution of system $(21)$.

We now prove the necessary condition. If system (21) has a unique periodic solution, then the subsystem (10) must have at least one periodic solution. Suppose that the subsystem (10) has two periodic solutions $\left(\tilde{S}_{a}(t), \tilde{I}_{a}(t)\right)$ and $\left(\phi_{1}(t), \phi_{2}(t)\right)$. Then $\left(\tilde{S}_{a}(t), \tilde{I}_{a}(t), \tilde{S}_{h}(t), \tilde{I}_{h}(t)\right)$ and $\left(\phi_{1}(t), \phi_{2}(t), \phi_{3}(t), \phi_{4}(t)\right)$ are periodic solutions of system (21), where

$$
\phi_{3}(t)=e^{-\beta_{h} \int_{t_{0}}^{t} \phi_{2}(s) d s-\mu_{h} t}\left(e^{\mu_{h} t_{0}} \phi_{3}^{*}\left(t_{0}\right)+\Pi_{h} \int_{t_{0}}^{t} e^{\beta_{h} \int_{t_{0}}^{s} \phi_{2}(u) d u} e^{\mu_{h} s} d s\right)
$$

and

$$
\phi_{4}(t)=e^{-\left(\mu_{h}+\delta_{h}+\gamma\right) t}\left(e^{\left(\mu_{h}+\delta_{h}+\gamma\right) t_{0}} \phi_{4}^{*}\left(t_{0}\right)+\beta_{h} \int_{t_{0}}^{t} e^{\left(\mu_{h}+\delta_{h}+\gamma\right) s} \phi_{2}(s) \phi_{3}(s) d s\right)
$$


tradiction. Therefore, the subsystem (10) has a unique limit cycle.

Theorem 3.10. If $m_{a}<\frac{\mu_{a}+\delta_{a}}{\beta_{a}}<\frac{M_{a}+m_{a}}{2}$, then the unique periodic solution $\left(\tilde{S}_{a}(t), \tilde{I}_{a}(t), \tilde{S}_{h}(t), \tilde{I}_{h}(t)\right)$ of the full system (21) is globally asymptotically stable if and only if the unique limit cycle $\left(\tilde{S}_{a}(t), \tilde{I}_{a}(t)\right)$ of the subsystem (10) is globally asymptotically stable.

Proof. The necessary condition is obvious. We only prove the sufficient condition.

By Theorem 3.5, the unique limit cycle $\Gamma$ of the subsystem (10) is globally asymptotically stable in $D_{2}$ if $m_{a}<\frac{\mu_{a}+\delta_{a}}{\beta_{a}}<\frac{M_{a}+m_{a}}{2}$. For any solution $\left(S_{a}(t), I_{a}(t)\right)$, by the results in Coppel [17] (p. 82) or Coddington and Levinson [14] (p. 323), we have

$$
\lim _{t \rightarrow \infty}\left|S_{a}(t)-\tilde{S}_{a}(t+c)\right|=0, \lim _{t \rightarrow \infty}\left|I_{a}(t)-\tilde{I}_{a}(t+c)\right|=0
$$

where $c$ is some constant depending on $\left(S_{a}(t), I_{a}(t)\right)$.

Next, we prove that $\lim _{t \rightarrow \infty}\left|S_{h}(t)-\tilde{S}_{h}(t+c)\right|=0$. Since

$$
\left|S_{h}(t)-\tilde{S}_{h}(t)\right|=e^{-\beta_{h} \int_{t_{0}}^{t} \tilde{I}_{a}(s) d s} e^{-\mu_{h}\left(t-t_{0}\right)}\left|S_{h}\left(t_{0}\right)-S_{h}^{*}\left(t_{0}\right)\right|
$$

we have

$$
\lim _{t \rightarrow \infty}\left|S_{h}(t)-\tilde{S}_{h}(t)\right|=0 .
$$

Since $\lim _{t \rightarrow \infty}\left|I_{a}(t)-\tilde{I}_{a}(t+c)\right|=0, \forall \varepsilon>0$, there exists a $T_{1}>0$ such that if $t>T_{1}$, then

$$
\tilde{I}_{a}(t+c)-\varepsilon<I_{a}(t)<\tilde{I}_{a}(t+c)+\varepsilon .
$$

We construct the following equations:

$$
\frac{d S_{h}^{-}}{d t}=\Pi_{h}-\beta_{h}\left(\tilde{I}_{a}(t+c)+\varepsilon\right) S_{h}-\mu_{h} S_{h},
$$




$$
\frac{d S_{h}^{+}}{d t}=\Pi_{h}-\beta_{h}\left(\tilde{I}_{a}(t+c)-\varepsilon\right) S_{h}-\mu_{h} S_{h} .
$$

The equation (29) has a unique periodic solution $\tilde{S}_{h}^{-}(t)$ and

$$
\lim _{t \rightarrow \infty}\left|S_{h}^{-}(t)-\tilde{S}_{h}^{-}(t)\right|=0
$$

The equation (30) has a unique periodic solution $\tilde{S}_{h}^{+}(t)$ and

$$
\lim _{t \rightarrow \infty}\left|S_{h}^{+}(t)-\tilde{S}_{h}^{+}(t)\right|=0
$$

where,

$$
\begin{aligned}
& \tilde{S}_{h}^{-}(t)=e^{-\beta_{h} \int_{t_{0}}^{t}\left(\tilde{I}_{a}(s+c)+\varepsilon\right) d s-\mu_{h} t}\left[e^{\mu_{h} t_{0}} S_{h}^{*}\left(t_{0}\right)+\Pi_{h} \int_{t_{0}}^{t} e^{\beta_{h} \int_{t_{0}}^{s}\left[\tilde{I}_{a}(u+c)+\varepsilon\right] d u} e^{\mu_{h} s} d s\right], \\
& \tilde{S}_{h}^{+}(t)=e^{-\beta_{h} \int_{t_{0}}^{t}\left[\tilde{I}_{a}(s+c)-\varepsilon\right] d s-\mu_{h} t}\left[e^{\mu_{h} t_{0}} S_{h}^{*}\left(t_{0}\right)+\Pi_{h} \int_{t_{0}}^{t} e^{\beta_{h} \int_{t_{0}}^{s}\left[\tilde{I}_{a}(u+c)-\varepsilon\right] d u} e^{\mu_{h} s} d s\right],
\end{aligned}
$$

$\tilde{S}_{h}^{-}(t)$ is defined on the three-dimensional cylinder $\Gamma^{-} \times \mathbb{R}^{+} \times \mathbb{R}^{+}=\Gamma^{-} \times[0, \infty) \times[0, \infty)$ and $\tilde{S}_{h}^{+}(t)$ is defined on the three-dimensional cylinder $\Gamma^{+} \times \mathbb{R}^{+} \times \mathbb{R}^{+}=\Gamma^{+} \times[0, \infty) \times[0, \infty)$, where $\Gamma^{-}=\left\{\left(\tilde{S}_{a}(t), \tilde{I}_{a}(t)+\varepsilon\right): t \in[0, \omega]\right\}$ and $\Gamma^{+}=\left\{\left(\tilde{S}_{a}(t), \tilde{I}_{a}(t)-\varepsilon\right): t \in[0, \omega]\right\}, \mathbb{R}^{+}=[0, \infty)$.

By the third equation of system (21) and the comparison theorem of ordinary differential equations, we have

$$
S_{h}^{-}(t)<S_{h}(t)<S_{h}^{+}(t)
$$

for $t>T_{1}$.

Next, we prove that

$$
\lim _{t \rightarrow \infty}\left|\tilde{S}_{h}^{-}(t)-\tilde{S}_{h}(t+c)\right|=0, \lim _{t \rightarrow \infty}\left|\tilde{S}_{h}^{+}(t)-\tilde{S}_{h}(t+c)\right|=0 .
$$

Since

$$
\frac{d S_{h}(t+c)}{d t}=\Pi_{h}-\beta_{h} I_{a}(t+c) S_{h}(t+c)-\mu_{h} S_{h}(t+c),
$$

we have,

$$
\tilde{S}_{h}(t+c)=e^{-\beta_{h} \int_{t_{0}}^{t} \tilde{I}_{a}(s+c) d s-\mu_{h} t}\left[e^{\mu_{h} t_{0}} S_{h}^{*}\left(t_{0}+c\right)+\Pi_{h} \int_{t_{0}}^{t} e^{\beta_{h} \int_{t_{0}}^{s} \tilde{I}_{a}(u+c) d u} e^{\mu_{h} s} d s\right] .
$$

Thus,

$$
\begin{aligned}
\tilde{S}_{h}^{-}(t)-\tilde{S}_{h}(t+c)= & e^{-\beta_{h} \int_{t_{0}}^{t} \tilde{I}_{a}(s+c) d s-\mu_{h}\left(t-t_{0}\right)}\left[e^{-\beta_{h} \varepsilon\left(t-t_{0}\right)} S_{h}^{-*}\left(t_{0}\right)-S_{h}^{*}\left(t_{0}+c\right)\right] \\
& +e^{-\beta_{h} \int_{t_{0}}^{t}\left(\tilde{I}_{a}(s+c)+\varepsilon\right) d s-\mu_{h} t} \int_{t_{0}}^{t} e^{\beta_{h} \int_{t_{0}}^{s} \tilde{I}_{a}(u+c) d u} e^{\mu_{h} s}\left[e^{\beta_{h} \varepsilon\left(s-t_{0}\right)}-1\right] d s, \\
\tilde{S}_{h}^{+}(t)-\tilde{S}_{h}(t+c)= & e^{-\beta_{h} \int_{t_{0}}^{t} \tilde{I}_{a}(s+c) d s-\mu_{h}\left(t-t_{0}\right)}\left[e^{-\beta_{h} \varepsilon\left(t-t_{0}\right)} S_{h}^{-*}\left(t_{0}\right)-S_{h}^{*}\left(t_{0}+c\right)\right] \\
& +e^{-\beta_{h} \int_{t_{0}}^{t}\left(\tilde{I}_{a}(s+c)-\varepsilon\right) d s-\mu_{h} t} \int_{t_{0}}^{t} e^{\beta_{h} \int_{t_{0}}^{s} \tilde{I}_{a}(u+c) d u} e^{\mu_{h} s}\left[e^{-\beta_{h} \varepsilon\left(s-t_{0}\right)}-1\right] d s .
\end{aligned}
$$

If $\varepsilon$ is small enough, then

$$
\lim _{t \rightarrow \infty}\left|\tilde{S}_{h}^{-}(t)-\tilde{S}_{h}(t+c)\right|=0, \lim _{t \rightarrow \infty}\left|\tilde{S}_{h}^{+}(t)-\tilde{S}_{h}(t+c)\right|=0 .
$$


By (31), we have

$$
S_{h}^{-}(t)-\tilde{S}_{h}(t+c)<S_{h}(t)-\tilde{S}_{h}(t+c)<S_{h}^{+}(t)-\tilde{S}_{h}(t+c)
$$

Since

$$
\begin{aligned}
& \left|S_{h}^{-}(t)-\tilde{S}_{h}(t+c)\right| \leq\left|S_{h}^{-}(t)-\tilde{S}_{h}^{-}(t)\right|+\left|\tilde{S}_{h}^{-}(t)-\tilde{S}_{h}(t+c)\right|, \\
& \left|S_{h}^{+}(t)-\tilde{S}_{h}(t+c)\right| \leq\left|S_{h}^{+}(t)-\tilde{S}_{h}^{+}(t)\right|+\left|\tilde{S}_{h}^{+}(t)-\tilde{S}_{h}(t+c)\right|,
\end{aligned}
$$

we have

$$
\lim _{t \rightarrow \infty}\left|S_{h}^{-}(t)-\tilde{S}_{h}(t+c)\right|=0, \lim _{t \rightarrow \infty}\left|S_{h}^{+}(t)-\tilde{S}_{h}(t+c)\right|=0 .
$$

Hence,

$$
\lim _{t \rightarrow \infty}\left|S_{h}(t)-\tilde{S}_{h}(t+c)\right|=0 .
$$

Similarly, we can prove $\lim _{t \rightarrow \infty}\left|I_{h}(t)-\tilde{I}_{h}(t+c)\right|=0$.

Let $F_{i}=\left\{\left(S_{a}, I_{a}, S_{h}, I_{h}, R_{h}\right) \mid\left(S_{a}, I_{a}\right) \in D_{i}, S_{h} \geq 0, I_{h} \geq 0, R_{h} \geq 0\right\}$ with $i=1,2$. Finally, we have the following results on the global dynamics of the original system (9) with Allee effect for the avain population.

Corollary 3.11. (i) The disease-free equilibrium $H_{1}\left(0,0, S_{h}^{*}, 0,0\right)$ of model (9) avian Allee effect is always globally asymptotically stable in $F_{1}$; (ii) if $\frac{\mu_{a}+\delta_{a}}{\beta_{a}} \geq M_{a}$, then the disease-free equilibrium $H_{3}\left(M_{a}, 0, S_{h}^{*}, 0,0\right)$ of model (9) avian Allee effect is globally asymptotically stable in $F_{2}$; (iii) if $\frac{M_{a}+m_{a}}{2} \leq \frac{\mu_{a}+\delta_{a}}{\beta_{a}}<M_{a}$, then the unique endemic equilibrium $H_{4}\left(S_{a}^{* *}, I_{a}^{* *}, S_{h}^{* *}, I_{h}^{* *}, R_{h}^{* *}\right)$ of model (9) avian Allee effect is globally asymptotically stable in $F_{2}$; (iv) if $m_{a}<\frac{\mu_{a}+\delta_{a}}{\beta_{a}}<\frac{M_{a}+m_{a}}{2}$, then there is a unique periodic solution of model (9) avian Allee effect at the neighborhood of the endemic equilibrium $H_{4}\left(S_{a}^{* *}, I_{a}^{* *}, S_{h}^{* *}, I_{h}^{* *}, R_{h}^{* *}\right)$ which is globally asymptotically stable in $F_{2}$.

\section{Numerical simulations}

\subsection{Comparison and numerical simulations of the basic reproduction numbers}

By the results in Sections 2 and 3, we know that the basic reproduction numbers of systems (4) and (9) are $\mathcal{R}_{0,1}=\frac{K_{a} \beta_{a}}{\mu_{a}+\delta_{a}}$ and $\mathcal{R}_{0,2}=\frac{\beta_{a}\left(M_{a}+m_{a}\right)\left(\mu_{a}+\delta_{a}\right)}{\left(\mu_{a}+\delta_{a}\right)^{2}+M_{a} m_{a} \beta_{a}^{2}}$, respectively. Now we keep the maximal carrying capacity in systems (4) and (9) identical (i.e., $K_{a}=M_{a}$ ), then we can easily obtain that

$$
\begin{aligned}
\mathcal{R}_{0,2}-\mathcal{R}_{0,1} & =\frac{\beta_{a}\left(M_{a}+m_{a}\right)\left(\mu_{a}+\delta_{a}\right)}{\left(\mu_{a}+\delta_{a}\right)^{2}+M_{a} m_{a} \beta_{a}^{2}}-\frac{\beta_{a} K_{a}}{\mu_{a}+\delta_{a}} \\
& =\frac{\beta_{a}\left(M_{a}+m_{a}\right)\left(\mu_{a}+\delta_{a}\right)}{\left(\mu_{a}+\delta_{a}\right)^{2}+M_{a} m_{a} \beta_{a}^{2}}-\frac{\beta_{a} M_{a}}{\mu_{a}+\delta_{a}} \\
& =\frac{\beta_{a} m_{a}\left(\mu_{a}+\delta_{a}+\beta_{a} M_{a}\right) \beta_{a}\left(\frac{\mu_{a}+\delta_{a}}{\beta_{a}}-M_{a}\right)}{\left(\mu_{a}+\delta_{a}\right)\left[\left(\mu_{a}+\delta_{a}\right)^{2}+M_{a} m_{a} \beta_{a}^{2}\right]} .
\end{aligned}
$$

Thus, we have the following results:

(i) If $K_{a}=M_{a}$ and $\frac{\mu_{a}+\delta_{a}}{\beta_{a}} \leq m_{a}$, then $\mathcal{R}_{0,1}>1 \geq \mathcal{R}_{0,2}$;

(ii) If $K_{a}=M_{a}$ and $m_{a}<\frac{\mu_{a}+\delta_{a}}{\beta_{a}}<M_{a}$, then $\mathcal{R}_{0,1}>\mathcal{R}_{0,2}>1$;

(iii) If $K_{a}=M_{a}$ and $\frac{\mu_{a}+\delta_{a}}{\beta_{a}}=M_{a}$, then $\mathcal{R}_{0,1}=\mathcal{R}_{0,2}=1$;

(iv) If $K_{a}=M_{a}$ and $\frac{\mu_{a}+\delta_{a}}{\beta_{a}}>M_{a}$, then $\mathcal{R}_{0,1}<\mathcal{R}_{0,2}<1$.

In order to substantiate the above results, we present some numerical simulations as follows. First we fix some parameters. We assume human and the wild avian can survive 70 years and 8 years, respectively. Hence the natural death rates of human and wild avian population are 


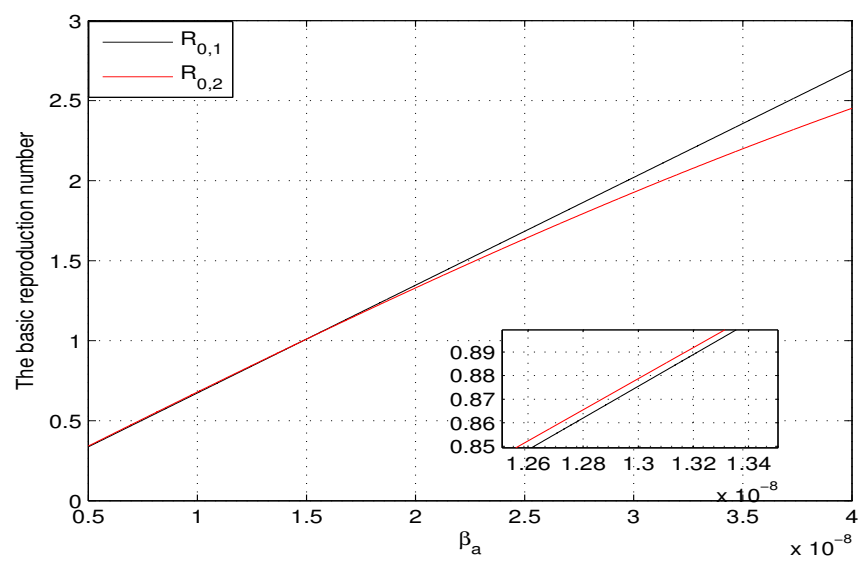

Figure 2: The plot shows the changes of $\mathcal{R}_{0,1}$ and $\mathcal{R}_{0,2}$ with respect to $\beta_{a}$.

$\mu_{h}=3.91 * 10^{-5}$ and $\mu_{a}=3.4246 * 10^{-4}$ per day, respectively. We also assume that the diseaserelated death rates of the infected avian and the infective human population are $\delta_{a}=4 * 10^{-4}$ and $\delta_{h}=0.3445$ per day, respectively; the intrinsic growth rate of the avian population is $r_{a}=5 * 10^{-3}$; the maximal and critical carrying capacities of the avian population are $K_{a}=M_{a}=50000$ and $m_{a}=800$, respectively. The numerical simulations of the basic reproduction numbers of both systems are given in Fig. 2.

From Fig. 2, we know that if $\beta_{a}<1 * 10^{-8}$, then $\mathcal{R}_{0,1}<\mathcal{R}_{0,2}<1$; if $\beta_{a}>2 * 10^{-8}$, then $\mathcal{R}_{0,1}>\mathcal{R}_{0,2}>1$; if $\beta_{a}=1.48492 * 10^{-8}$, then $\mathcal{R}_{0,1}=\mathcal{R}_{0,2}=1$. These results support the theoretical conclusions.

\subsection{Numerical simulations of the models}

Noted that the expression $\frac{\mu_{a}+\delta_{a}}{\beta_{a}}$ is a key quantity. The relationship of size between $\frac{\mu_{a}+\delta_{a}}{\beta_{a}}$ and $K_{a}$ or $m_{a}, M_{a}$ determines whether the avian influenza disappears or not. When $\mu_{a}, \delta_{a}, m_{a}$ and $M_{a}$ are fixed, then $\beta_{a}$ is a key parameter. In this subsection, we investigate the influence of parameter $\beta_{a}$ on the number of infected humans by performing some numerical simulations. Besides the fixed parameters in the above subsection, we further assume that the recovery rate of infectious human individuals is 0.1 per day, so $\gamma=0.1$. In general, avian influenza mainly outbreaks in a specific location. We estimate that the number of susceptible avian population is between 100000 and 1000000 , the number of infective avian population is between 0 and 100 , and the number of susceptible human population is between 100000 and 1000000 in the region. So we choose the initial values as $\left(S_{a}(0), I_{a}(0), S_{h}(0), I_{h}(0), R_{h}(0)\right)=(100000,100,100000,1,0)$.

Firstly, we study the influence of parameter $\beta_{a}$ on the number of infective individuals of model (4) with logistic avian growth. When parameters $K_{a}, \mu_{a}$, and $\delta_{a}$ are fixed, the threshold value $\beta_{a}^{*}=1.48492 * 10^{-8}$ such that $\mathcal{R}_{0,1}=1$. If $\beta_{a} \leq \beta_{a}^{*}$, the disease disappears and the solution $I_{h}(t)$ is asymptotically stable and converges to the disease-free state value (see Fig. 3(A)); If $\beta_{a}>\beta_{a}^{*}$, the endemic disease is prevalent, the solution $I_{h}(t)$ is asymptotically stable and converges to the endemic state value (see Fig. 3(B)). Furthermore, we can also observe that the peak value of $I_{h}(t)$ increases with $\beta_{a}$ increasing from Fig. 3 .

Secondly, we investigate the influence of parameter $\beta_{a}$ on the number of infective individuals of model (9) with avian Allee effect. Recall that $\mathcal{R}_{0,2}=1 \Leftrightarrow \frac{\mu_{a}+\delta_{a}}{\beta_{a}}=m_{a}$ or $\frac{\mu_{a}+\delta_{a}}{\beta_{a}}=M_{a}$. Then for fixed parameters $\mu_{a}, \delta_{a}, m_{a}$ and $M_{a}$, the threshold value $\beta_{a}^{*}=1.48492 * 10^{-8}$ or $9.28075 * 10^{-7}$ such that $\mathcal{R}_{0,2}=1$. According to Corollary 3.11 , for the above parameter and initial values, if $\beta_{a} \geq 9.28075 * 10^{-7}$, the disease disappears and the solution $I_{h}(t)$ is asymptotically stable in the region $F_{2}$ (see Fig. $4(\mathrm{~A})$ ); if $2.9231 * 10^{-8} \leq \beta_{a}<9.28075 * 10^{-7}$, the endemic disease is prevalent, the solution $I_{h}(t)$ is asymptotically stable in the region $F_{2}$ (see Fig. $4(\mathrm{~B})$ ). 
Thirdly, we simulate the periodic solutions of model (9) with avian Allee effect. Parameters $\mu_{a}, \delta_{a}, \mu_{h}, \gamma, M_{a}, m_{a}$ and $r_{a}$ are chosen as before. Other parameters and initial values are selected as follows: $\Pi_{h}=30, \beta_{h}=6 * 10^{-8}, \delta_{h}=0.3445,\left(S_{a}(0), I_{a}(0), S_{h}(0), I_{h}(0), R_{h}(0)\right)=$ $(1000000,2000,100000,30,5)$. When $\beta_{a}=2.57 * 10^{-7}$ or $\beta_{a}=2.58 * 10^{-7}$, then $\frac{\mu_{a}+\delta_{a}}{\beta_{a}}$ is between $m_{a}$ and $\frac{M_{a}+m_{a}}{2}$, which satisfies the condition of Corollary 3.11. Hence, there is a unique periodic solution of system (9) in the neighborhood of the endemic equilibrium which is globally asymptotically stable in $F_{2}$ (see Fig. $4(\mathrm{C})$ ).


Figure 3: The plots display the changes of $I_{h}(t)$ with $\beta_{a}$ varying where $\beta_{h}=6 * 10^{-9}$. (A) Solutions $I_{h}(t)$ are asymptotically stable and converge to the disease-free state value; (B) Solutions $I_{h}(t)$ are asymptotically stable and converges to the endemic state value.
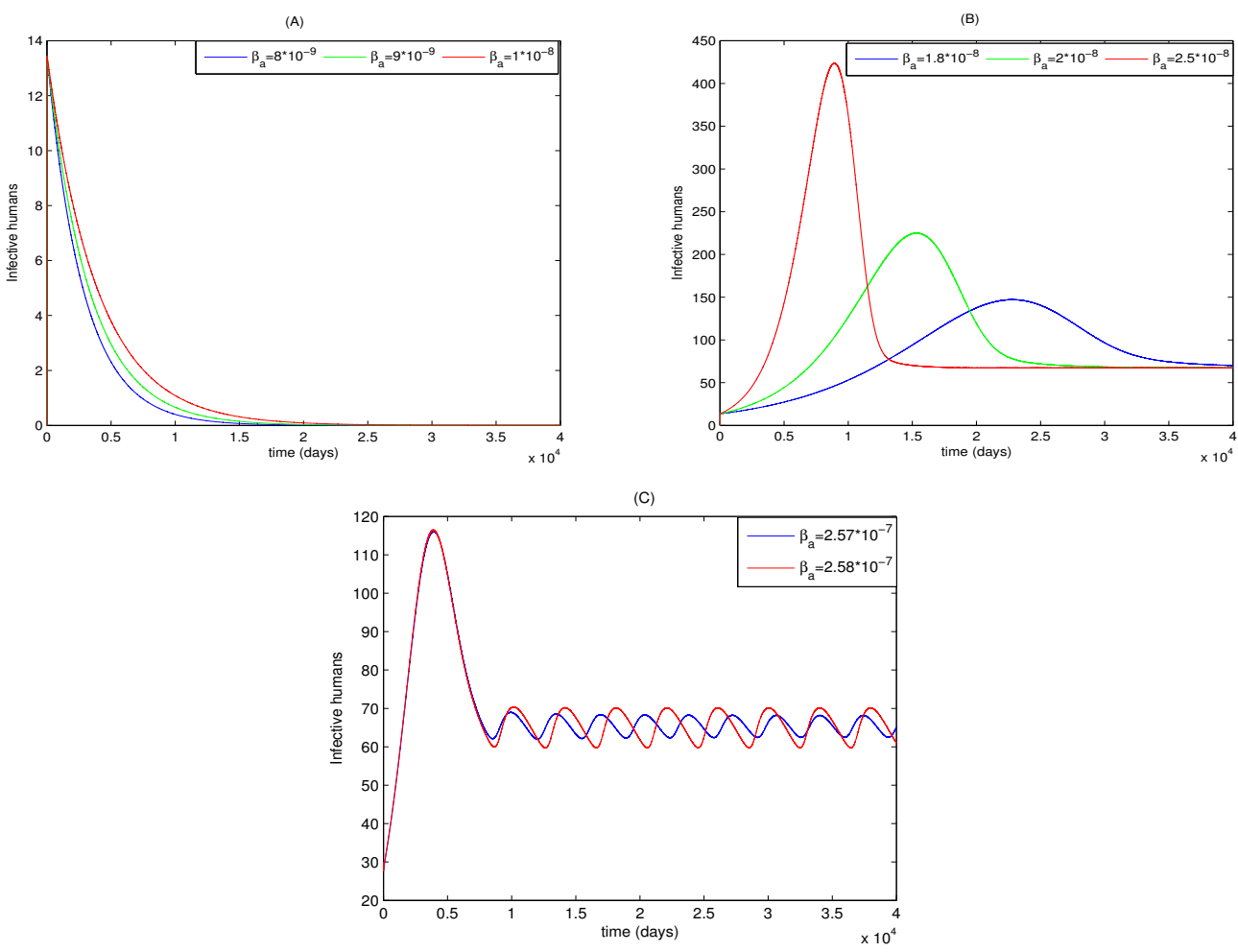

Figure 4: The plots bring to light the changes of $I_{h}(t)$ with $\beta_{a}$ varying. (A) $I_{h}(t)$ is asymptotically stable in the region $F_{2}$ and converges to the disease-free state value where the disease-free equilibrium is $(50000,0,767263.43,0,0)$; (B) $I_{h}(t)$ is asymptotically stable in the region $F_{2}$ and converges to the endemic state value; (C) The periodic solution $I_{h}(t)$ is asymptotically stable in $F_{2}$.

Finally, we examine the influence of parameter $\beta_{h}$ on the number of infective individuals of model (4) with logistic avian growth and model (9) with avian Allee effect. When the birds are at 
endemic state, we can observe that the human population is also at endemic state even if bird-tohuman contact rate $\left(\beta_{h}\right)$ is reduced by $99 \%$ (see Fig. 5). Furthermore, we can also observe that the peak value of $I_{h}(t)$ and the endemic state value of these systems increase when $\beta_{h}$ is increasing (see Fig. 5).
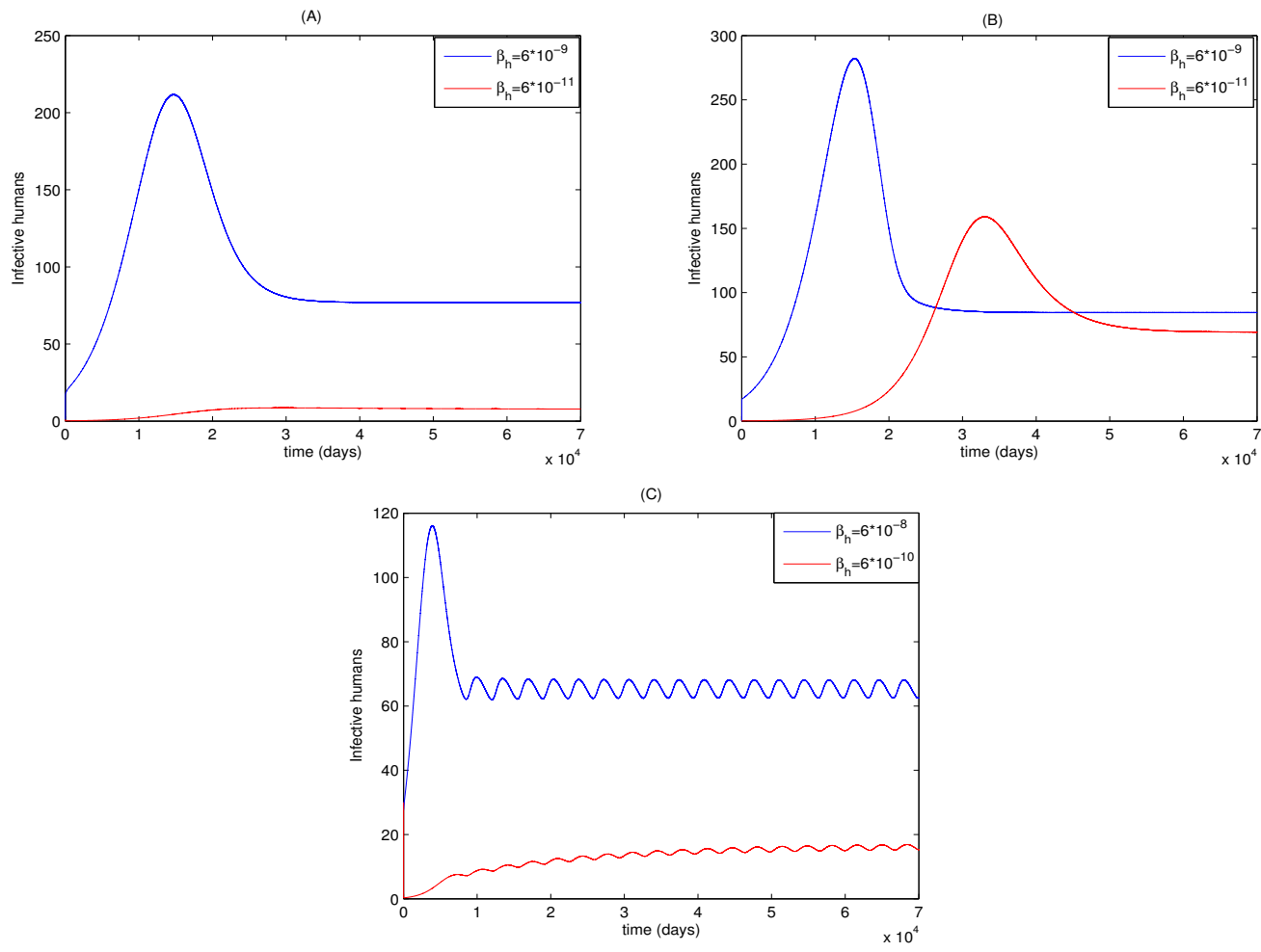

Figure 5: The plots reveal the changes of $I_{h}(t)$ with $\beta_{h}$ varying. (A) $I_{h}(t)$ of system (4) with avian logistic growth is asymptotically stable and converges to the endemic state value; (B) $I_{h}(t)$ of system (9) with avian Allee effect converges to the endemic state value; $(\mathrm{C})$ The periodic solution $I_{h}(t)$ of system (9) with avian Allee effect is asymptotically stable.

\section{Discussion}

It is believed that the H7N9 was transferred to ducks in China by wild birds through migration along the East Asian flyway (Liu et al. [40]). Experimental data (Pantin-Jackwood et al. [46]) showed that it is conceivable that passerine birds may serve as vectors for transmission of H7N9 virus to domestic poultry (Jones et al. [32]), which in turn transmitted the virus to humans through live-poultry markets (Bao et al. [4], Chen et al. [11]). After the first outbreak in the spring of 2013, the H7N9 avian influenza resurged in China from November 2013 to May 2014 and again from November 2014 to June 2015 (WHO [58]). The data strongly indicate that it is becoming seasonal and persistent like the H5N1 avian influenza. Tuncer and Martcheva [52] used periodic contact/incidence rates to model the seasonality in H5N1 avian influenza transmission. Since the live-poultry markets are open all year around, the contact/incidence rates are more likely to be constant in this case. Cross-sectional surveys conducted in China after the outbreaks of the avian influenza A H7N9 viruses show a high degree of awareness of human avian influenza in both urban and rural populations, a higher level of proper hygienic practice among urban residents, and in particular a dramatically reduced number of visits to live markets in urban population after the H7N9 outbreak in 2013. Taking into account the psychological effect toward avian influenza in the human population, we (Liu et al. [41]) proposed a bird-to-human transmission model in which the avian population exhibits saturation effect. However, our study shows that the saturation effect within avian population and the psychological effect in human population cannot change the 
stability of equilibria but can affect the number of infected humans if the disease is prevalent, so there is no periodic solutions. In Liu et al. [42], we also took account of the incubation periods of avian influenza A virus, constructed a bird-to-human transmission model with different time delays in the avian and human populations combining the survival probability of the infective avian and human populations at the latent time, and obtained global asymptotical stability of equilibria of the system. Once again the time delays in such models do not induce oscillations. Chen et al. [10] argued that the lack of understanding of the virus ecology in birds has resulted in the persistent circulating of H7N9 in China. Since the H7N9 virus does not induce clinical signs in poultry and is classified as a low pathogenicity avian influenza virus (Pantin-Jackwood et al. [46]), we believe that the population dynamics of avian species contribute significantly to the persistence and potential periodicity of the virus in avian as well as human populations. Note that it has been observed (Serrano et al. [49]) the growth of some avian populations exhibit Allee effect due to habitat destruction, spread of alien species, pollution, and diseases.

In this paper, to study the transmission dynamics of avian influenza from birds to humans we constructed ordinary differential equation models with two different growth laws for the avian population: (i) logistic growth and (ii) Allee effect. We obtained a threshold value for the prevalence of avian influenza and discussed the local or global asymptotical stability of each equilibrium of these systems. Our results indicate that the asymptotic dynamics of the model with logistic growth for the avian population are completely determined by the basic reproduction number: the disease-free equilibrium exists and is locally asymptotically stable if the basic reproduction number is less than the unity; the disease-free equilibrium becomes unstable and the endemic equilibrium exists and is locally asymptotically stable if the basic reproduction number is greater than the unity. Global asymptotic stability of these equilibria were also established by using Liapunov function method and LaSalle's invariance principle. For the model with Allee effect for the avian population, beside stability results it was shown that periodic solutions exists via Hopf bifurcations. Global stability of the periodic solutions was also considered.

Recall that for the system (4) with logistic avian growth, the basic reproduction number was given as follows

$$
\mathcal{R}_{0,1}=\frac{K_{a} \beta_{a}}{\mu_{a}+\delta_{a}} .
$$

There were two disease-free equilibria given by $A\left(0,0, S_{h}^{*}, 0,0\right)$ and $B\left(K_{a}, 0, S_{h}^{*}, 0,0\right)$, where $S_{h}^{*}=$ $\frac{\Pi_{h}}{\mu_{h}}$. If $\mathcal{R}_{0,1}>1$, and a unique endemic equilibrium given by $C\left(S_{a}^{* *}, I_{a}^{* *}, S_{h}^{* *}, I_{h}^{* *}, R_{h}^{* *}\right)$, where

$$
\begin{aligned}
& S_{a}^{* *}=\frac{\mu_{a}+\delta_{a}}{\beta_{a}}, I_{a}^{* *}=\frac{r_{a}\left(\mu_{a}+\delta_{a}\right)}{K_{a} \beta_{a}^{2}}\left(\mathcal{R}_{0,1}-1\right), \\
& S_{h}^{* *}=\frac{\Pi_{h}}{\beta_{h} I_{a}^{* *}+\mu_{h}}, I_{h}^{* *}=\frac{\beta_{h} I_{a}^{* *} S_{h}^{* *}}{\mu_{h}+\delta_{h}+\gamma}, R_{h}^{* *}=\frac{\gamma I_{h}^{* *}}{\mu_{h}} .
\end{aligned}
$$

We only consider the biologically meanful equilibria $B\left(K_{a}, 0, S_{h}^{*}, 0,0\right)$ and $C\left(S_{a}^{* *}, I_{a}^{* *}, S_{h}^{* *}, I_{h}^{* *}, R_{h}^{* *}\right)$, the results about system (4) with logistic avian growth can be summarized in the following chart $(\mathrm{BRN}=$ basic reproduction number).

Table 1: Stability chart for system (4) with logistic avian growth

\begin{tabular}{||c|c|c|c|}
\hline Conditions & BRN & $B\left(K_{a}, 0, S_{h}^{*}, 0,0\right)$ & $C\left(S_{a}^{* *}, I_{a}^{* *}, S_{h}^{* *}, I_{h}^{* *}, R_{h}^{* *}\right)$ \\
\hline$\frac{\mu_{a}+\delta_{a}}{\beta_{a}}>K_{a}$ & $\mathcal{R}_{0,1}<1$ & globally stable & does not exist \\
\hline$\frac{\mu_{a}+\delta_{a}}{\beta_{a}}<K_{a}$ & $\mathcal{R}_{0,1}>1$ & unstable & globally stable \\
\hline
\end{tabular}

For the system (9) with Allee effect in the avian population, the basic reproduction number is given by

$$
\mathcal{R}_{0,2}=\frac{\beta_{a}\left(M_{a}+m_{a}\right)\left(\mu_{a}+\delta_{a}\right)}{\left(\mu_{a}+\delta_{a}\right)^{2}+M_{a} m_{a} \beta_{a}^{2}} .
$$


There are three disease-free equilibria given by $H_{1}\left(0,0, S_{h}^{*}, 0,0\right), H_{2}\left(m_{a}, 0, S_{h}^{*}, 0,0\right)$, and $H_{3}\left(M_{a}, 0, S_{h}^{*}\right.$, $0,0)$, where $S_{h}^{*}=\frac{\Pi_{h}}{\mu_{h}}$, and if $\mathcal{R}_{0,2}>1$, there is also a unique endemic equilibrium given by $H_{4}\left(S_{a}^{* *}, I_{a}^{* *}, S_{h}^{* *}, I_{h}^{* *}, R_{h}^{* *}\right)$, where

$$
\begin{aligned}
& S_{a}^{* *}=\frac{\mu_{a}+\delta_{a}}{\beta_{a}}, I_{a}^{* *}=\frac{r_{a}}{\beta_{a}} \frac{\beta_{a}^{2} M_{a} m_{a}+\left(\mu_{a}+\delta_{a}\right)^{2}}{M_{a} m_{a} \beta_{a}^{2}}\left(\mathcal{R}_{0,2}-1\right), \\
& S_{h}^{* *}=\frac{\Pi_{h}}{\beta_{h} I_{a}^{* *}+\mu_{h}}, I_{h}^{* *}=\frac{\beta_{h} I_{a}^{* *} S_{h}^{* *}}{\mu_{h}+\delta_{h}+\gamma}, R_{h}^{* *}=\frac{\gamma I_{h}^{* *}}{\mu_{h}} .
\end{aligned}
$$

Similarly, considering only the biologically meaningful equilibria we can summarize the results about system (9) with Allee effect in the avian population in the following chart (GSPS=globally stable periodic solution).

Table 2: Stability chart for system (9) with Allee effect in the avian population

\begin{tabular}{||c|c|c|c|c|}
\hline Conditions & BRN & $H_{2}\left(m_{a}, 0, S_{h}^{*}, 0,0\right)$ & $H_{4}\left(S_{a}^{* *}, I_{a}^{* *}, S_{h}^{* *}, I_{h}^{* *}, R_{h}^{* *}\right)$ & $H_{3}\left(M_{a}, 0, S_{h}^{*}, 0,0\right)$ \\
\hline$M_{a}<\frac{\mu_{a}+\delta_{a}}{\beta_{a}}$ & $\mathcal{R}_{0,2}<1$ & unstable & does not exists & globally stable \\
\hline$\frac{m_{a}+M_{a}}{2}<\frac{\mu_{a}+\delta_{a}}{\beta_{a}}<M_{a}$ & $\mathcal{R}_{0,2}>1$ & unstable & globally stable & unstable \\
\hline$m_{a}<\frac{\mu_{a}+\delta_{a}}{\beta_{a}}<\frac{m_{a}+M_{a}}{2}$ & $\mathcal{R}_{0,2}>1$ & unstable & unstable (GSPS) & unstable \\
\hline$\frac{\mu_{a}+\delta_{a}}{\beta_{a}}<m_{a}$ & $\mathcal{R}_{0,2}<1$ & unstable & does not exist & unstable \\
\hline
\end{tabular}

Through the analysis, we found that if the maximal carrying capacity of the avian population of each system is the same (i.e., $K_{a}=M_{a}$ ) and $m_{a}<\frac{\mu_{a}+\delta_{a}}{\beta_{a}}<M_{a}$, then $\mathcal{R}_{0,1}>\mathcal{R}_{0,2}>1$, which indicates that the transmission speed of the avian influenza virus of system (4) (with logistic growth) is greater than system (9) (with Allee effect) and the endemic disease of the two systems is prevalent; if the maximal carrying capacity of each system is the same and $\frac{\mu_{a}+\delta_{a}}{\beta_{a}} \leq m_{a}$, then $\mathcal{R}_{0,1}>1 \geq \mathcal{R}_{0,2}$, which indicates that the endemic disease of system (4) is prevalent but the endemic disease of system (9) disappears; if the maximal carrying capacity of each system is the same and $\frac{\mu_{a}+\delta_{a}}{\beta_{a}}>M_{a}$, then $\mathcal{R}_{0,1}<\mathcal{R}_{0,2}<1$, which indicates that the endemic disease of both systems disappears. Therefore, we can make the quantity $\frac{\mu_{a}+\delta_{a}}{\beta_{a}}$ greater than the maximal carrying capacity of the avian population to control the disease by reducing $\beta_{a}$ (transmission rate from infective avian to susceptible avian) or increasing $\mu_{a}$ (natural death rate of the avian population) and $\delta_{a}$ (disease-related death rate of the infected avian). The effective methods will be to reduce the transmission between the susceptible and infective avian populations and isolating or culling the infective birds if necessary.

For the system (4) with logistic avian growth, from Table 1 we can see that if $\frac{\mu_{a}+\delta_{a}}{\beta_{a}}>K_{a}$ so that $\mathcal{R}_{0,1}<1$, then the disease-free equilibrium $B\left(K_{a}, 0, S_{h}^{*}, 0,0\right)$ is globally stable; if $\frac{\mu_{a}+\delta_{a}}{\beta_{a}}<K_{a}$ so that $\mathcal{R}_{0,1}>1$, then the disease-free equilibrium $B\left(K_{a}, 0, S_{h}^{*}, 0,0\right)$ becomes unstable and the endemic equilibrium $C\left(S_{a}^{* *}, I_{a}^{* *}, S_{h}^{* *}, I_{h}^{* *}, R_{h}^{* *}\right)$ exists and is globally stable. For the system (9) with Allee effect in the avian population, the dynamics are more interesting. If $M_{a}<\frac{\mu_{a}+\delta_{a}}{\beta_{a}}$ (where $M_{a}$ is the maximal carrying capacity of the avian population) so that $\mathcal{R}_{0,2}<1$, then the disease-free equilibrium $H_{2}\left(m_{a}, 0, S_{h}^{*}, 0,0\right)$ with less avian density (where $m_{a}$ is the critical carry capacity of the avian population, $m_{a}<M_{a}$ ) is unstable and the disease-free equilibrium $H_{3}\left(M_{a}, 0, S_{h}^{*}, 0,0\right)$ with more avian density is globally stable; if $\beta_{a}$ increases or $\mu_{a}+\delta_{a}$ increases such that $\frac{m_{a}+M_{a}}{2}<\frac{\mu_{a}+\delta_{a}}{\beta_{a}}<M_{a}$ so $\mathcal{R}_{0,2}>1$, then the disease-free equilibrium $H_{3}\left(M_{a}, 0, S_{h}^{*}, 0,0\right)$ with more avian density becomes unstable and an endemic equilibrium $H_{4}\left(S_{a}^{* *}, I_{a}^{* *}, S_{h}^{* *}, I_{h}^{* *}, R_{h}^{* *}\right)$ exists and is globally stable; if $m_{a}<\frac{\mu_{a}+\delta_{a}}{\beta_{a}}<\frac{m_{a}+M_{a}}{2}$ so $\mathcal{R}_{0,2}>1$ remains hold, then the endemic equilibrium $H_{4}\left(S_{a}^{* *}, I_{a}^{* *}, S_{h}^{* *}, I_{h}^{* *}, R_{h}^{* *}\right)$ becomes unstable and there is a globally stable periodic orbit bifurcated from it; if, further, $\frac{\mu_{a}+\delta_{a}}{\beta_{a}}<m_{a}$ so that $\mathcal{R}_{0,2}<1$, both disease-free equilibria 
$H_{2}\left(m_{a}, 0, S_{h}^{*}, 0,0\right)$ and $H_{3}\left(M_{a}, 0, S_{h}^{*}, 0,0\right)$ exist and the disease die out. We have provided references to support the observation that the H7N9 avian virus has been transmitted from wild birds to domestic poultry and then to humans and pointed out some potential avian species that are believed to be responsible for the cross-species transmission. Though we are not able to obtain data on specific avian species and apply our models and conclusions directly, we believe that our results on the existence and stability of periodic solutions in the model with Allee effect for the avian population may be useful in understanding the seasonal/periodic outbreaks of the H7N9 avian influenza.

From the expressions of the basic reproduction numbers $\mathcal{R}_{0,1}$ and $\mathcal{R}_{0,2}$ defined in (32) and (35), respectively, and the existence and stability conditions listed in Tables 1 and 2, it seems that the parameters involving human population do not appear and the overall disease could be controlled if it can be controlled in birds. Theoretically it is true: if there is no disease among birds then there is no outbreaks in humans since there is no human-to-human transmission yet. However, H7N9 is classified as a low pathogenicity avian influenza virus and causes no symptoms and mortality in birds. Controlling the disease in the avian population is very difficult and the basic reproduction numbers do not provide effective control measures for the human population. Notice that $\beta_{h}$ (the transmission rate from infective avian to susceptible human) appears in the expressions (34) and (37) for the steady state values of $I_{h}^{* *}$, the number of infective human individuals. In fact, it should be understood that $\beta_{h}=c_{h} p_{h}$, where $c_{h}$ is the contact rate between a susceptible human and an infective bird and $p_{h}$ is the probability of transmitting the virus per contact. Thus, to prevent spread of the avian influenza virus from birds to humans, we suggest to reduce contacting poultry and to take extra protection when contacting is necessary. If either $c_{h}=0$ or $p_{h}=0$, then $I_{h}^{* *}=0$ and there is no outbreaks in humans. This also explains that in the spring of 2013, when the poultry markets in Jiangsu, Shanghai, and Zhejiang were temporarily closed, the outbreak was controlled soon.

Our study also indicates that if birds are at endemic state, then the human population is also at endemic state even if the bird-to-human contact rate $\left(\beta_{h}\right)$ is reduced by $99 \%$ (see Fig. 5). Furthermore, we can see that the peak value of $I_{h}(t)$ and the endemic state value of these systems increase when $\beta_{h}$ is increasing (see Fig. 5). Our models results may not accurately describe all situations, but they can explain most of situations because perfect prevention (i.e. $100 \%$ reduction of $\beta_{h}$ ) is unlikely to happen in reality.

Note that asymptotic dynamics of avian influenza models consisted of bird and human populations, in particular global stability in such models, have been studied by other researchers, see for example Iwami et al. [28] and Gumel [24]. Constant growth was assumed for the avian population in these studies. Compared to their models and results, our main contributions are as follows: First, we assumed that the growth rate of the avian population follows either the logistic law or the Allee effect, which is more general than the constant growth rate. Secondly, we not only obtained global stability of the disease-free and endemic equilibria but also established the global stability of the periodic solutions generated via Hopf bifurcations. To the best of our knowledge, there are very few results on the global stability of periodic solutions for epidemic models. Thus, our techniques could be useful to study the existence and global stability of periodic solutions in similar ecological and epidemiological models.

The roles of wild birds and domestic birds in the transmission of the H5N1 avian influenza are different and mathematical models have been proposed to include both types of birds (Bourouiba et al. [5], Gourley et al. [22], Lucchetti et al. [43], Tuncer and Martcheva [52]). It will be very interesting to include both wild birds and domestic birds in modeling the bird-to-human transmission of the H7N9 avian influenza, we are considering such a model, estimating model parameters, and trying to simulate the datasets on reported human H7N9 cases from China. The results will be reported somewhere else in the future.

Since the H7N9 virus is classified as a low pathogenicity avian influenza virus (LPAIV) (PantinJackwood et al. [46]), we ignored the recovery class of birds in our models. The model of Vaidya and Wahl [53] predicts that birds infected by avian influenza virus lose their immunity in approximately 
4 weeks, it would be interesting to take account of the recovery class of birds in future models of avian influenza.

Acknowledgement. The authors would like to thank Dr. Lan Zou and the anonymous reviewers for their helpful comments and suggestions.

\section{Appendices}

In this section, we prove the global stability of the full system (4) with logistic avian growth and the full system (9) with avian Allee effect by using LaSalle's invariance principle.

\subsection{Boundedness of solutions}

For system (4) with logistic avian growth, we have the following result.

Lemma 6.1. All solutions of system (4) with initial values in $\mathbb{R}_{+}^{5}$ are bounded.

Proof. Define a function $\eta=S_{a}+I_{a}+S_{h}+I_{h}+R_{h}$, then for each $\nu: 0<\nu<\min \left\{\mu_{a}, \mu_{h}\right\}$, the following inequality holds:

$$
\frac{d \eta}{d t}+\nu \eta \leq \frac{K_{a}\left(r_{a}+\nu\right)^{2}}{4 r_{a}}+\Pi_{h}=\phi .
$$

Applying the theory of differential inequalities ( [38]), we obtain that

$$
0<\eta\left(S_{a}, I_{a}, S_{h}, I_{h}, R_{h}\right)(t)<\frac{\phi}{\nu}\left(1-e^{-\nu t}\right)+\eta\left(S_{a}(0), I_{a}(0), S_{h}(0), I_{h}(0), R_{h}(0)\right) e^{-\nu t},
$$

and for $t \rightarrow \infty$ we have $0<\eta<\frac{\phi}{\nu}$.

For $\epsilon=1$, there exists $t_{0}>0$, if $t>t_{0}$ then $\left(S_{a}+I_{a}+S_{h}+I_{h}+R_{h}\right)(t)<\frac{\phi}{\nu}+1$. Furthermore, $\left(S_{a}+I_{a}+S_{h}+I_{h}+R_{h}\right)(t)$ is continuous on the interval $\left[0, t_{0}\right]$, so $\left(S_{a}+I_{a}+S_{h}+I_{h}+R_{h}\right)(t)$ has a maximum value $A^{*}$ on the interval $\left[0, t_{0}\right]$. Choose $M=\max \left\{A^{*}, \frac{\phi}{\nu}+1\right\}$, then $\left(S_{a}+I_{a}+S_{h}+\right.$ $\left.I_{h}+R_{h}\right)(t) \leq M$. Hence all the solutions of system (4) with initial values in $\mathbb{R}_{+}^{5}$ are confined in the region $D=\left\{\left(S_{a}, I_{a}, S_{h}, I_{h}, R_{h}\right) \in \mathbb{R}_{+}^{5}: S_{a}+I_{a}+S_{h}+I_{h}+R_{h} \leq M\right\}$.

Similarly, for system (9) with avian Alle effect, we have the following result.

Lemma 6.2. All solutions of system (9) with initial values in $\mathbb{R}_{+}^{5}$ are uniformly bounded in the region $F=\left\{\left(S_{a}, I_{a}, S_{h}, I_{h}, R_{h}\right) \in \mathbb{R}_{+}^{5}: S_{a}+I_{a}+S_{h}+I_{h}+R_{h} \leq \max \left\{A_{0}, \frac{\rho}{\omega}+1\right\}\right\}$, where $\omega: 0<\omega<$ $\min \left\{r_{a}, \mu_{a}+\delta_{a}, \mu_{h}\right\}, \rho=\frac{4 r_{a}\left(M_{a}+m_{a}\right)^{3}}{27 M_{a} m_{a}}+\Pi_{h}, A_{0}$ is the maximum value of $\left(S_{a}+I_{a}+S_{h}+I_{h}+R_{h}\right)(t)$ on interval $\left[0, t_{1}\right]$.

Proof. The proof is similar to that of Lemma 6.1, we omit it.

\subsection{Another proof of Theorem 2.6}

Proof. (i) According to Lemma 2.3, the disease-free equilibrium $B_{a}$ of system (5) is globally asymptotically stable if $\mathcal{R}_{0,1} \leq 1$ which implies that $S_{a} \rightarrow K_{a}$ and $I_{h} \rightarrow 0$ if $t \rightarrow \infty$. Hence, we analyze the global stability of $B_{a h}$ only at the region $D_{01}=\left\{\left(S_{a}, I_{a}, S_{h}, I_{h}\right) \mid S_{a}=K_{a}, I_{a}=0, S_{a}+I_{a}+S_{h}+I_{h} \leq\right.$ $M\}$. Consider system (6) with the avian components already at the disease-free steady state, given by

$$
\left\{\begin{array}{l}
\frac{d S_{h}}{d t}=\Pi_{h}-\mu_{h} S_{h} \\
\frac{d I_{h}}{d t}=-\left(\mu_{h}+\delta_{h}+\gamma\right) I_{h} .
\end{array}\right.
$$


Choose a Liapunov function as follows

$$
V_{21}=S_{h}-S_{h}^{*}-S_{h}^{*} \ln \frac{S_{h}}{S_{h}^{*}}+I_{h}
$$

then,

$$
\left.\frac{d V_{21}}{d t}\right|_{(38)}=\frac{S_{h}-S_{h}^{*}}{S_{h}}\left(\Pi_{h}-\mu_{h} S_{h}\right)-\left(\mu_{h}+\delta_{h}+\gamma\right) I_{h}=-\frac{\mu_{h}}{S_{h}}\left(S_{h}-S_{h}^{*}\right)^{2}-\left(\mu_{h}+\delta_{h}+\gamma\right) I_{h} \leq 0 .
$$

Since $D_{01}=\left\{\left(S_{a}, I_{a}, S_{h}, I_{h}\right) \mid S_{a}=K_{a}, I_{a}=0, S_{a}+I_{a}+S_{h}+I_{h} \leq M: \frac{d V_{21}}{d t}=0\right\}=\left\{\left(S_{a}, I_{a}, S_{h}, I_{h}\right)\right.$ : $\left.S_{a}=K_{a}, I_{a}=0, S_{h}=S_{h}^{*}, I_{h}=0\right\}=\left\{B_{a h}\right\}$, according to LaSalle's invariance principle (Hale [25]), the equilibrium $B_{a h}$ is globally asymptotically stable for positive trajectories.

(ii) Similarly, by Lemma 2.3, the endemic equilibrium $C_{a}$ of system (5) is globally asymptotically stable if $\mathcal{R}_{0,1}>1$ which shows that $S_{a} \rightarrow S_{a}^{* *}$ and $I_{a} \rightarrow I_{a}^{* *}$ if $t \rightarrow \infty$. We consider the global stability of $C_{a h}$ only at the region $D_{02}=\left\{\left(S_{a}, I_{a}, S_{h}, I_{h}\right) \mid S_{a}=S_{a}^{* *}, I_{a}=I_{a}^{* *}, S_{a}+I_{a}+S_{h}+I_{h} \leq M\right\}$. Consider system (6) with the avian components already at the endemic steady state, given by

$$
\left\{\begin{array}{l}
\frac{d S_{h}}{d t}=\Pi_{h}-\beta_{h} I_{a}^{* *} S_{h}-\mu_{h} S_{h} \\
\frac{d I_{h}}{d t}=\beta_{h} I_{a}^{* *} S_{h}-\left(\mu_{h}+\delta_{h}+\gamma\right) I_{h}
\end{array}\right.
$$

Choose the following Liapunov function

$$
V_{22}=S_{h}^{* *}\left(\frac{S_{h}}{S_{h}^{* *}}-\ln \frac{S_{h}}{S_{h}^{* *}}\right)+I_{h}^{* *}\left(\frac{I_{h}}{I_{h}^{* *}}-\ln \frac{I_{h}}{I_{h}^{* *}}\right),
$$

According to the proof of Theorem 2.6(ii), we have $\left.\frac{d V_{22}}{d t}\right|_{(39)} \leq 0$. Due to $D_{02}=\left\{\left(S_{a}, I_{a}, S_{h}, I_{h}\right) \mid S_{a}=\right.$ $\left.S_{a}^{* *}, I_{a}=I_{a}^{* *}, S_{a}+I_{a}+S_{h}+I_{h} \leq M: \frac{d V_{22}}{d t}=0\right\}=\left\{\left(S_{a}^{* *}, I_{a}^{* *}, S_{h}^{* *}, I_{h}^{* *}\right)\right\}=\left\{C_{a h}\right\}$, by the LaSalle's invariance principle, the endemic equilibrium $C_{a h}$ is globally asymptotically stable.

\subsection{Another proof of Theorem 3.8}

Set $E_{1}=\left\{\left(S_{a}, I_{a}, S_{h}, I_{h}\right):\left(S_{a}, I_{a}\right) \in D_{1}, S_{a}+I_{a}+S_{h}+I_{h} \leq \max \left\{A_{0}, \frac{\rho}{\omega}+1\right\}\right\}$ and $E_{2}=$ $\left\{\left(S_{a}, I_{a}, S_{h}, I_{h}\right):\left(S_{a}, I_{a}\right) \in D_{2}, S_{a}+I_{a}+S_{h}+I_{h} \leq \max \left\{A_{0}, \frac{\rho}{\omega}+1\right\}\right\}$, where $D_{1}$ and $D_{2}$ are defined in Theorem 3.5.

Proof. (i) If $\left(S_{a}, I_{a}, S_{h}, I_{h}\right) \in E_{1}$, then $\left(S_{a}, I_{a}\right) \in D_{1}$. According to Theorem 3.5, the disease-free equilibrium $O$ of the avian-only subsystem (10) is always globally asymptotically stable in the region $D_{1}$ which implies that $S_{a} \rightarrow 0$ and $I_{a} \rightarrow 0$ if $t \rightarrow \infty$. So we only consider the global stability of $O_{a h}$ only at the region $E_{12}=\left\{\left(S_{a}, I_{a}, S_{h}, I_{h}\right) \mid S_{a}=0, I_{a}=0, S_{a}+I_{a}+S_{h}+I_{h} \leq \max \left\{A_{0}, \frac{\rho}{\omega}+1\right\}\right\}$. Now consider system (21) with the avian components already at the disease-free steady state, given by

$$
\left\{\begin{array}{l}
\frac{d S_{h}}{d t}=\Pi_{h}-\mu_{h} S_{h} \\
\frac{d I_{h}}{d t}=-\left(\mu_{h}+\delta_{h}+\gamma\right) I_{h} .
\end{array}\right.
$$

Choose a Liapunov function as follows

$$
V_{31}=S_{h}-S_{h}^{*}-S_{h}^{*} \ln \frac{S_{h}}{S_{h}^{*}}+I_{h}
$$

According to the proof of Theorem 2.6(i), we have $E_{12}=\left\{\left(S_{a}, I_{a}, S_{h}, I_{h}\right) \mid S_{a}=K_{a}, I_{a}=0, S_{a}+I_{a}+\right.$ $\left.S_{h}+I_{h} \leq \max \left\{\frac{\phi}{\nu}+1, A_{0}\right\}: \frac{d V_{31}}{d t}=0\right\}=\left\{\left(S_{a}, I_{a}, S_{h}, I_{h}\right): S_{a}=0, I_{a}=0, S_{h}=S_{h}^{*}, I_{h}=0\right\}=\left\{O_{a h}\right\}$, 
LaSalle's invariance principle (Hale [25]) implies that the equilibrium $O_{a h}$ is globally asymptotically stable for positive trajectories in the region $E_{1}$.

(ii) If $\frac{M_{a}+m_{a}}{2} \leq \frac{\mu_{a}+\delta_{a}}{\beta_{a}}<M_{a}$ and $\left(S_{a}, I_{a}, S_{h}, I_{h}\right) \in E_{2}$, then $\left(S_{a}, I_{a}\right) \in D_{2}$. According to Theorem 3.5, the disease-free equilibrium $E$ of the subsystem (10) is always globally asymptotically stable in the region $D_{2}$ which shows that $S_{a} \rightarrow S_{a}^{* *}$ and $I_{h} \rightarrow I_{a}^{* *}$ if $t \rightarrow \infty$. Thus we only need to analyze the global stability of $E_{a h}$ only at the region $E_{22}=\left\{\left(S_{a}, I_{a}, S_{h}, I_{h}\right) \mid S_{a}=S_{a}^{* *}, I_{a}=\right.$ $\left.I_{a}^{* *}, S_{a}+I_{a}+S_{h}+I_{h} \leq \max \left\{A_{0}, \frac{\rho}{\omega}+1\right\}\right\}$. Once again consider system (21) with the avian components already at the disease-free steady state, given by

$$
\left\{\begin{array}{l}
\frac{d S_{h}}{d t}=\Pi_{h}-\beta_{a} I_{a}^{* *} S_{h}-\mu_{h} S_{h} \\
\frac{d I_{h}}{d t}=\beta_{a} I_{a}^{* *} S_{h}-\left(\mu_{h}+\delta_{h}+\gamma\right) I_{h} .
\end{array}\right.
$$

Choose the following Liapunov function

$$
V_{32}=S_{h}^{* *}\left(\frac{S_{h}}{S_{h}^{* *}}-\ln \frac{S_{h}}{S_{h}^{* *}}\right)+I_{h}^{* *}\left(\frac{I_{h}}{I_{h}^{* *}}-\ln \frac{I_{h}}{I_{h}^{* *}}\right),
$$

According to the proof of Theorem 2.6(ii), we have $E_{22}=\left\{\left(S_{a}, I_{a}, S_{h}, I_{h}\right) \mid S_{a}=S_{a}^{* *}, I_{a}=I_{a}^{* *}, S_{a}+\right.$ $\left.I_{a}+S_{h}+I_{h} \leq \max \left\{A_{0}, \frac{\rho}{\omega}+1\right\}: \frac{d V_{32}}{d t}=0\right\}=\left\{\left(S_{a}, I_{a}, S_{h}, I_{h}\right): S_{a}=S_{a}^{* *}, I_{a}=I_{a}^{* *}, S_{h}=S_{h}^{* *}, I_{h}=\right.$ $\left.I_{h}^{* *}\right\}=\left\{E_{a h}\right\}$, LaSalle's invariance principle then implies that the equilibrium $E_{a h}$ is globally asymptotically stable for positive trajectories in the region $E_{2}$.

(iii) If $\frac{\mu_{a}+\delta_{a}}{\beta_{a}} \geq M_{a}$ and $\left(S_{a}, I_{a}, S_{h}, I_{h}\right) \in E_{2}$, then $\left(S_{a}, I_{a}\right) \in D_{2}$. By Theorem 3.5 , the diseasefree equilibrium $B$ of the subsystem (10) is globally asymptotically stable in the region $D_{2}$ which illustrates that $S_{a} \rightarrow M_{a}$ and $I_{h} \rightarrow 0$ if $t \rightarrow \infty$. Similarly we only need to study the global stability of $B_{a h}$ only at the region $E_{22}=\left\{\left(S_{a}, I_{a}, S_{h}, I_{h}\right) \mid S_{a}=M_{a}, I_{a}=0, S_{a}+I_{a}+S_{h}+I_{h} \leq\right.$ $\left.\max \left\{A_{0}, \frac{\rho}{\omega}+1\right\}\right\}$. To do so we consider system (21) with the avian components already at the disease-free steady state, given by

$$
\left\{\begin{array}{l}
\frac{d S_{h}}{d t}=\Pi_{h}-\mu_{h} S_{h} \\
\frac{d I_{h}}{d t}=-\left(\mu_{h}+\delta_{h}+\gamma\right) I_{h} .
\end{array}\right.
$$

Choose a Liapunov function as follows

$$
V_{33}=S_{h}-S_{h}^{*}-S_{h}^{*} \ln \frac{S_{h}}{S_{h}^{*}}+I_{h}
$$

Proceeding with the proof process of (i), we have $E_{22}=\left\{\left(S_{a}, I_{a}, S_{h}, I_{h}\right) \mid S_{a}=M_{a}, I_{a}=0, S_{a}+I_{a}+\right.$ $\left.S_{h}+I_{h} \leq \max \left\{A_{0}, \frac{\rho}{\omega}+1\right\}: \frac{d V_{33}}{d t}=0\right\}=\left\{\left(S_{a}, I_{a}, S_{h}, I_{h}\right): S_{a}=M_{a}, I_{a}=0, S_{h}=S_{h}^{*}, I_{h}=0\right\}=$ $\left\{B_{a h}\right\}$, by LaSalle's invariance principle we claim that the equilibrium $B_{a h}$ is globally asymptotically stable for positive trajectories in the region $E_{2}$.

\section{References}

[1] S. Altizer, R. Bartel, and B. A. Han, Animal migration and infectious disease risk, Science 331 (2011), 296-302.

[2] W. C. Allee, Animal Aggregation: A Study in General Sociology, University of Chicago Press, Chicago, 1931.

[3] R. M. Anderson and R. M. May, Infectious Diseases of Humans: Dynamics and Control, Oxford University Press, Oxford, 1991. 
[4] C. Bao, L. Cui, M. Zhou, L. Hong, H. Wang et al. Live-animal markets and influenza A (H7N9) virus infection, New Eng. J. Med. 368 (2013), 2337-2339.

[5] L. Bourouiba, S. A. Gourley, R. Liu and J. Wu, The interaction of migratory birds and domestic poultry and its role in sustaining avian influenza, SIAM J. Appl. Math. 71 (2011), 487-516.

[6] R. Burrows, H. Hofer and M. L. East, Population dynamics, intervention and survival in African wild dogs (Lycaon pictus), Proc. R. Soc. B 262 (1995), 235-245.

[7] L. Cai, G. Chen and D. Xiao, Multiparametric bifurcations of an epidemiological model with strong Allee effect, J. Math. Biol. 67 (2013), 185-215.

[8] Centers for Disease Control and Prevention (CDC), Types of influenza virus, Retrieved January 15, 2014. http://www.cdc.gov/flu/about/viruses/types.htm.

[9] Center for Infectious Disease Research and Policy (CIDRAP), China reports three H7N9 infections, two fatal (April 1, 2013). http://www.cidrap.umn.edu/newsperspective/2013/04/china-reports-three-h7n9-infections-two-fatal.

[10] E. Chen, Y. Chen, L. Fu, Z. Chen, Z. Gong et al., Human infection with avian influenza A(H7N9) virus re-emerges in China in winter 2013, Euro Surveill. 18 (2013), 43.

[11] Y. Chen, W. Liang, S. Yang, N. Wu, H. Gao et al., Human infections with the emerging avian influenza A H7N9 virus from wet market poultry: clinical analysis and characterisation of viral genome, Lancet 381 (2013), 1916-1925.

[12] N. S. Chong and R. J. Smith, Modeling avian influenza using Filippov systems to determine culling of infected birds and quarantine, Nonlinear Anal. Real World Appl. 24 (2015), 196-218.

[13] D. L. Clifford, J. A. K. Mazet, E. J. Dubovi, D. K. Garcelon, T. J. Coonan et al., Pathogen exposure in endangered island fox (Urocyon littoralis) populations: implications for conservation management, Biol. Conserv. 131 (2006), 230-243.

[14] E. A. Coddington and N. Levinson, Theory of Ordinary Differential Equations, McGraw-Hill, New York, 1955.

[15] W. A. Coppel, Quadratic systems with a degenerate critical point, Bull. Austral. Math. Soc. 38 (1988), 1-10.

[16] W. A. Coppel, A new class of quadratic systems, J. Differential Equations 92 (1991), 360-372.

[17] W. A. Coppel, Stability of Asymptotic Behavior of Differential Equations, Heath, Boston, 1965.

[18] F. de Castro and B. Bolker, Mechanisms of disease induced extinction, Ecol. Lett. 8 (2005), $117-126$.

[19] O. Diekmann, J. A. P. Heesterbeek and M. G. Roberts, The construction of next generation matrices for compartmental epidemic models, J. R. Soc. Interface 7 (2000), 873-885.

[20] A. Friedman and A.-A. Yakubu, Fatal disease and demographic Allee effect: population persistence and extinction, J. Biol. Dyn. 6 (2012), 495-508.

[21] A. Friedman and A.-A. Yakubu, Host demographic Allee effect, fatal disease, and migration: persistence or extinction, SIAM J. Appl. Math. 72 (2012), 1644-1666.

[22] S. A. Gourley, R. Liu and and J. Wu, Spatiotemporal distributions of migratory birds: Patchy models with delay, SIAM J. Appl. Dyn. Syst. 9 (2010), 589-610. 
[23] J. Guckenheimer and P. Holmes, Nonlinear Oscillation, Dynamical Systems and Bifurcation of Vector Fields, Springer-Verlag, New York, 1983.

[24] A. B. Gumel, Global dynamics of a two-strain avian influenza model, Intl. J. Comput. Math. 86 (2009), 85-108.

[25] J. K. Hale, Ordinary Differential Equations, Wiley, New York, 1969.

[26] F. M. Hilker, M. Langlais and H. Malchow, The Allee effect and infectious diseases: extinction, multistability and the (dis-)appearance of oscillations, Am. Nat. 173 (2009), 72-88.

[27] F. M. Hilker, M. Langlais, S.V. Petrovskii and H. Malchow, A diffusive SI model with Allee effect and application to FLV, Math. Biosci. 206 (2007), 61-80.

[28] S. Iwami, Y. Takeuchi and X. Liu, Avian-human influenza epidemic model, Math. Biosci. 207 (2007), 1-25.

[29] S. Iwami, Y. Takeuchi and X. Liu, Avian flu pandemic: Can we prevent it? J. Theor. Biol. 257 (2009), 181-190.

[30] S. Iwami, Y. Takeuchi, X. Liu and S. Nakaoka. A geographical spread of vaccine-resistance in avian influenza epidemics, J. Theor. Biol. 259 (2009), 219-228.

[31] J. C. Jones, S. Sonnberg, Z. A. Kocer, K. Shanmuganatham, P. Seiler et al., Possible role of songbirds and parakeets in transmission of influenza A(H7N9) virus to humans, Emerg. Infect. Dis. 20 (2014), 380-385.

[32] J. C. Jones, S. Sonnberg, R. J. Webby and R. G. Webster, Influenza A (H7N9) virus transmission between finches and poultry, Emerg. Infect. Dis. 21 (2015), 619-628.

[33] E. Jung, S. Iwami, Y. Takeuchi and T.-C. Jo, Optimal control strategy for prevention of avian influenza pandemic, J. Theor. Biol. 260 (2009), 220-229.

[34] Y. Kang and C. Castillo-Chavez, A simple epidemiological model for populations in the wild with Allee effects and disease-modified fitness, Discrete Contin. Dyn. Syst. B 19 (2014), 89130.

[35] Y. Kang and C. Castillo-Chavez, Dynamics of SI models with both horizontal and vertical transmissions as well as Allee effects, Math. Biosci. 248 (2014), 97-116.

[36] M. J. Keeling and P. Rohani, Modeling Infectious Diseases in Humans and Animals, Princeton University Press, Princeton, 2008.

[37] M. Koopmans and M. D. De Jong, Avian influenza A H7N9 in Zhejiang, China, Lancet 381 (2013), 1882-1883.

[38] V. Lakshmikantham, S. Leela, and A.A. Martynyuk, Stability Analysis of Nonlinear Systems, Marcel Dekker Inc., New York/Basel, 1989.

[39] Q. Li, L. Zhou, M. Zhou, Z. Chen, F. Li et al., Epidemiology of human infections with avian influenza A (H7N9) virus in China, New Eng. J. Med. 370 (2014), 520-532.

[40] D. Liu, W. Shi, Y. Shi, D. Wang, H. Xiao et al., Origin and diversity of novel avian influenza A H7N9 viruses causing human infection: phylogenetic, structural, and coalescent analyses, Lancet 381 (2013), 1926-1932.

[41] S. Liu, L. Pang, S. Ruan and X. Zhang, Global dynamics of avian influenza epidemic models with psychological effect, Comput. Math. Methods Med. 2015, Article ID 913726, 12 pages. http://dx.doi.org/10.1155/2015/913726. 
[42] S. Liu, S. Ruan and X. Zhang, On avian influenza epidemic models with time delay, Theory Biosci. 134 (2015), 75-82.

[43] J. Lucchetti, M. Roy and M. Martcheva, An avian influenza model and its fit to human avian influenza cases, in "Advances in Disease Epidemiology" (J. M. Tchuenche, Z. Mukandavire, Eds.), Nova Science Publishers, New York, 2009, pp. 1-30.

[44] X. Ma and W. Wang, A discrete model of avian influenza with seasonal reproduction and transmission, J. Biol. Dyn. 4 (2010), 296-314.

[45] National Health and Family Planning Commission of China (NHFPC), National Notifiable Disease Situation, http://en.nhfpc.gov.cn/diseases.html (in English); http://www.nhfpc.gov.cn/zhuzhan/yqxx/lists.shtml (in Chinese).

[46] M. J. Pantin-Jackwood, P. J. Miller, E. Spackman, D. E. Swayne, L. Susta, M. Costa-Hurtado and D. L. Suarez, Role of poultry in the spread of novel H7N9 influenza virus in China, $J$. Virol. 88 (2014), 5381-5390.

[47] J. H. Rappole and Z. Hubalek, Migratory birds and West Nile virus, J. Appl. Microbiol. 94 (2003), 47S-58S.

[48] J. C. Senar and M. J. Conroy, Multi-state analysis of the impacts of avian pox on a population of Serins (Serinus serinus): the importance of estimating recapture rates, Anim. Biodivers. Conserv. 27 (2004), 1-15.

[49] D. Serrano, D. Oro, E. Urua and J. Tella, Colony size selection and determines adult survival and dispersal preference: Allee effect in a colonial birds, Am. Nat. 166 (2005) (2), E22-E31.

[50] S. K. Skagen and A. A. A. Yackel, Potential misuse of avian density as a conservation metric, Conserv. Biol. 25 (2011), 48-55.

[51] H. R. Thieme, T. Dhirasakdanon, Z. Han and R. Trevino, Species decline and extinction: synergy of infectious disease and Allee effect?, J Biol. Dyn. 3 (2009), 305-323.

[52] N. Tuncer and M. Martcheva, Modeling seasonality in avian influenza H5N1, J. Biol. Syst. 21 (2013) (4), 1340004, 1-30.

[53] N. K. Vaidya and L. M. Wahl, Avian influenza dynamics under periodic environmental conditions, SIAM J. Appl. Math. 75 (2015) (2), 443-467.

[54] P. van den Driessche and J. Watmough, Reproduction numbers and sub-threshold endemic equilibria for compartmental models of disease transmission, Math. Biosci. 180 (2002), 29-48.

[55] X.-S. Wang and J. Wu, Periodic systems of delay differential equations and avian influenza dynamics, J. Math. Sci. 201 (2014), 693-704.

[56] M. Wikelski, J. Foufopoulos, H. Vargas and H. Snell, Galápagos birds and diseases: invasive pathogens as threats for island species, Ecol. Soc. 9 (2004): 5.

[57] D. S. Wilcove, D. Rothstein, J. Dubow, A. Phillips and E. Losos, Quantifying threats to imperiled species in the United States. BioScience 48 (1998), 607-615.

[58] World Health Organization (WHO), Human infection with avian influenza A(H7N9) virus - update (February 24, 2014). http://www.who.int/csr/don/2014_02_24/en/.

[59] World Organization for Animal Health (OIE), OIE expert mission finds live bird markets play a key role in poultry and human infections with influenza A (H7N9). Paris (April 30, 2013). http://www.oie.int/en/for-the-media/press-releases/detail/article/oie-expert-missionfinds-live-bird-markets-play-a-key-role-in-poultry-and-human-infections-with-infl/ 
[60] J. Zhang and B. Feng, The Geometric Theory and Bifurcation Problems of Ordinary Differential Equations, Peking University Press, Beijing, 1997 (in Chinese).

[61] Z. Zhang, T. Ding, W. Huang and Z. Dong, Qualitative Theory of Differential Equations, Science Press, Beijing, 1985 (in Chinese). English Ed., Transl. Math. Monographs Vol. 101, Amer. Math. Soc., Providence, RI, 1992.

[62] J. Zhang, Z. Jin, G. Sun, X. Sun, Y. Wang and B. Huang, Determination of original infection source of H7N9 avian influenza by dynamical model, Sci. Rep. 4 (2014), 1-16. 